\title{
Entropia Topológica e Aplicações à Teoria de Nós
}

\author{
Maria Alice Bertolim ${ }^{1}$
}

Orientador: Prof. Dr. Oziride Manzoli Neto

Dissertação apresentada ao Instituto de Ciências Matemáticas e de Computação da Universidade de São Paulo, como parte dos requisitos para obtenção do título de "Mestre em Ciências - Área de Matemática".

\author{
USP - São Carlos \\ Janeiro - 1999
}

\footnotetext{
${ }^{1}$ Este trabalho teve suporte financeiro do CNPq
} 
"Para tudo há um tempo, para cada coisa há um momento debaixo dos céus..." Ecle. 3,1 
À minha grande familia 


\section{Agradecimentos}

Agradecer é uma tarefa difícil... É chegado o momento de lembrar das pessoas que de alguma forma contribuíram para que eu tivesse condiçōes de chegar até aqui.

São muitas as pessoas que compartilharam esses momentos, mas agradeço em especial:

Ao Dide (Prof. Dr. Oziride Manzoli Neto) pela orientação, dedicação, incentivo, paciência, compreensão, e acima de tudo pela amizade, uma pessoa que sem dúvida mais que um orientador foi um grande amigo durante esse periodo.

Aos meus pais, Casemiro e Santina, meus irmãos Edson e Wilson, minhas cunhadas Shirlei e Mirian, minha avó Maria, enfim, à toda a minha família que mesmo distantes demonstraram seu apoio, carinho e compreenderam a minha ausência. Amo vocês!

Aos professores e funcionários da E.E.P.S.G. "Ferdinando Ienny" de Ouro Verde onde iniciei os meus estudos.

Ao pessoal do Centro Cultural de Ouro Verde, com quem trabalhei por muitos anos.

Aos professores e funcionários da UNESP de Presidente Prudente pelo incentivo para que eu continuasse os estudos e aos amigos que lá encontrei, em especial à Régia e Pedro Henrique, que jamais os esquecerei.

Aos professores do ICMC-USP, em especial à Ires, Washington, Maria Aparecida, Plácido, A. Conde, Biasi e Maria do Carmo, com os quais muito aprendi, e aos funcionários deste mesmo Instituto, em especial à Beth, Laura e Marília, pela atenção.

À Priscila pelos ensinamentos de Inglês.

Agradeço do fundo do coração à minha "segunda familia": Luciana, Fernando e Vera, e aos amigos: Eliane, Márcio, José, Ana Cláudia, Alexandra, Regilene, Marcão, Aninha, José Hilário, Luis, Claudemir, Cláudia, Marcelo Polezzi, Rogério, Daniela, Kátia, Marta, Pedro, Victor, Monica, Miguel, Daniela Rebolho, Andréa, ... pelo convívio e amizade mesmo nos momentos em que conviver comigo foi tarefa difícil.

Aos pequenos: Gabriela, Felipe, Alessa e Mayna que sempre sorriram pra mim e tornaram esses dias mais bonitos.

As pessoas que direta ou indiretamente colaboraram com esse trabalho.

Agradeço enfim a Deus por estar presente a cada dia e por todas as oportunidades. 


\section{Resumo}

Nesta dissertação apresentamos com bastante detalhes e exemplos a noção de entropia topológica e razão de crescimento exponencial, bem como relações entre estes conceitos.

Baseados nestas noçōes é definida a entropia de um nó e apresentadas algumas propriedades deste invariante. 


\begin{abstract}
In this work we present the notion of topological entropy and the exponential growth rate as well as the relations between these concepts with many details and examples.

Based on these notions the entropy of a knot is defined and some properties of this invariant are presented.
\end{abstract}




\section{Sumário}

$\begin{array}{ll}\text { Introdução } & 1\end{array}$

1 Preliminares 3

2 Entropia Topológica 12

2.1 Definições e Propriedades Gerais . . . . . . . . . . . . . . . 12

2.2 Teoremas Gerais . . . . . . . . . . . . . . . . . 21

2.3 Cálculo de entropia em espaços métricos $\ldots \ldots \ldots \ldots \ldots$

3 Entropia em Espaços Métricos e Razão de Crescimento Exponencial 42

3.1 Entropia em Espaços Métricos . . . . . . . . . . . . . . . . 42

3.2 Razão de Crescimento Exponencial . . . . . . . . . . . . 48

4 Entropia de Nós 53

$\begin{array}{ll}\text { Referências Bibliográficas } & 67\end{array}$ 


\section{Introdução}

A possibilidade de um estudo matemático de nós provavelmente foi primeiro reconhecida por K. F. Gauss. Suas investigações de eletrodinâmica (1833) juntamente com uma formulação analítica do número de enlaçamentos, foram ferramentas básicas para a teoria de nós e outros ramos da topologia.

A primeira tentativa da classificação de nós foi feita por um grupo inglês cerca de 50 anos mais tarde. $O$ desenvolvimento de nós e enlaçamentos teve que esperar pelo desenvolvimento da topologia e topologia algébrica, iniciado por $\mathrm{H}$. Poincaré por perto da virada do século. Por sua vez, a teoria de nós proporciona considerável estímulo para o desenvolvimento de muitas idéias importantes na topologia algébrica, teoria de grupos e outros campos.

Recentemente, a teoria de nós tem atraído interesses renovados devido ao grande progresso da teoria em dimensões altas, bem como novas aplicaçōes.

O estudo de nós baseia-se fortemente na obtenção de invariantes.

O objetivo principal deste trabalho é o estudo detalhado de um invariante de $n o ́, h_{K}$, chamado entropia do nó.

No capítulo 1 daremos definições básicas e alguns resultados necessários para o desenvolvimento do trabalho. A principal referência deste capítulo é o livro "Knots and Links" de D. Rolfsen.

No capítulo 2 introduziremos a noção de entropia como um invariante para funçōes contínuas. Consideraremos espaços topológicos compactos e todas as definições, propriedades e teoremas, serão feitos em função de coberturas. No final do capítulo daremos exemplos do cálculo de entropia em espaços métricos, 
utilizando a definição dada por meio de coberturas. A referência básica deste capítulo é o artigo "Topological Entropy" de R. L. Adler, A. G. Konheim and M. H. McAndrew.

No capítulo 3 definiremos entropia topológica por meio de medidas, para conjuntos bem distribuídos e separados. Estudaremos também quando a definiçāo dada no capítulo anterior coincide com a atual definição e daremos alguns exemplos do cálculo de entropia a fim de que haja um maior entendimento de suas propriedades. Também será definida a razão de crescimento exponencial $(E G R(\alpha))$ de um endomorfismo $\alpha$ de um grupo, preparando para uma análise de como EGR pode estimar a entropia de um nó $\left(h_{K}\right)$ que será feita no capítulo 4 . Este capítulo tem como referência básica o artigo "Knot invariants from topological Entropy" de Daniel S. Silver.

No capítulo 4 definiremos a entropia de um nó $\left(h_{K}\right)$. Relacionaremos EGR $\operatorname{com} h_{K}$, apresentando dois teoremas que não serāo demonstrados por envolverem resultados fortes de homeomorfismo pseudo-Anosov. Apresentaremos também uma forma de definir entropia para nós não fibrados. A partir do conceito de nós satélites definimos um sistema reduzido de nó. Assumiremos mais alguns resultados de homeomorfismo pseudo-Anosov para demonstrar o Teorema 4.0.3. A referência básica também foi o artigo "Knot invariants from topological Entropy" de Daniel S. Silver. 


\section{Capítulo 1}

\section{Preliminares}

Este capítulo contém notaçōes básicas, definiçōes e alguns resultados básicos necessários nos capítulos posteriores e que serão apresentados sem demonstração. Todas as variedades consideradas serão diferenciáveis $\left(C^{\infty}\right)$ ou terão estrutura "Piecewise Linear" (PL).

Definição 1.0.1. Seja $X$ um espaço topológico cuja homologia é zero a partir de um certo $n_{0} \in \mathbb{N}$. A característica de Euler $\chi(X)$ é definida por

$$
\chi(X)=\sum_{i=0}^{n_{0}}(-1)^{i}\left(\operatorname{rank} H_{i}(X)\right)
$$

onde $H_{i}(X)$ é o i-ésimo grupo de homologia de $X$.

Proposição 1.0.1. (Teorema da Classificação de Superfícies Orientadas) [20] Toda superfície conexa, orientada, compacta e sem bordo é homeomorfa à esfera ou à soma conexa de toros $\left(S^{1} \times S^{1}\right)$ (número finito). Duas superfícies compactas, conexas, orientadas e com bordo serão homeomorfas se o número de componentes de bordo forem o mesmo e suas superfícies sem bordo associadas forem homeomorfas. 
Definição 1.0.2. O número de toros $\left(S^{1} \times S^{1}\right)$ como na Proposição 1.0.1 é chamado genus da superfície.

Observação 1.0.1. A tabela abaixo mostra as superfícies, seus genus e características de Euler.

\begin{tabular}{|c|c|c|c|c|c|}
\hline superficie & $S^{2}$ & $T^{2}$ & $T^{2} \sharp T^{2}$ & $\ldots$ & $T^{2} \sharp \ldots \sharp T^{2}$ \\
\hline genus & 0 & 1 & 2 & $\ldots$ & $g$ \\
\hline$\chi$ & 2 & 0 & -2 & $\therefore$ & $2-2 g$ \\
\hline
\end{tabular}

Definição 1.0.3. Um toro sólido $V$ é um espaço homeomorfo a $S^{1} \times D^{2}$. Um homeomorfismo fixado $f: S^{1} \times D^{2} \rightarrow V$ é chamado um framing de $V$.

Proposição 1.0.2. [20] Seja $V$ um toro sólido com bordo $\partial V$ e $J$ uma curva fechada simples essencial (não homotopicamente trivial) em $\partial V$. Então são equivalentes as condições:

(a) J é homologicamente trivial em $V$ ( $V=$ toro sólido),

(b) J é homotopicamente trivial em $V$,

(c) J borda um disco $D$ em $V$,

(d) para algum framing $f: S^{1} \times D^{2} \rightarrow V, J=f\left(\{1\} \times \partial D^{2}\right)$.

Definiçāo 1.0.4. Uma curva simples fechada em $\partial V$ satisfazendo as condições da Proposição 1.0.2 é chamada um meridiano de V. Uma longitude de $V$ é qualquer curva fechada simples em $\partial V$ da forma $f\left(S^{1} \times\{1\}\right)$, para algum framing $f$ de $V$. 
Definiçāo 1.0.5. Um nó em um espaço topológico $X$ é um subespaço $K \subset$ $X$ homeomorfo a alguma esfera $S^{p}$. Um enlaçamento é um subespaço de $X$ homeomorfo à uma reunião disjunta (finita) de esferas $S_{1}^{p_{1}} \cup \ldots \cup S_{r}^{p_{r}}$.

Dois nós ou enlaçamentos $K_{1}$ e $K_{2}$ em $X$ são equivalentes se existe um homeomorfismo $f: X \rightarrow X$ tal que $f\left(K_{1}\right)=K_{2}$; neste caso usaremos a notação $\left(X, K_{1}\right) \cong\left(X, K_{2}\right)$. Outras definições de equivalência também aparecem na literatura, por exemplo: Se $f_{1}: S^{k} \rightarrow X$ e $f_{2}: S^{k} \rightarrow X$ são mergulhos de $S^{k}$ que definem $K_{1}$ e $K_{2}$, podemos definir que $f_{1}$ e $f_{2}$ são equivalentes se forem isotópicos. No caso de enlaçamentos de duas ou mais componentes podemos determinar uma ordem fixa das componentes e exigir que $f$ respeite esta ordem. A classe de equivalência de um nó ou enlaçamento é chamada seu tipo de nó ou tipo de enlaçamento. (Na maioria das vezes tomaremos $X=\mathbb{R}^{n}$ ou $X=S^{n}$ ). Muitas vezes olhamos para o nó como sendo $K \subset X$, outras vezes olhamos para o mergulho $f: S^{k} \rightarrow X$ que o define.

Podemos considerar $S^{3}$ como $S^{1} * S^{1}$ (join). Os pontos da forma $\left(x, y, \frac{1}{2}\right)$ constituem um toro $T\left(\approx S^{1} \times S^{1}\right)$ em $S^{3}$. Dados $p$ e $q$ primos entre si, consideremos o mergulho $T_{p, q}: S^{1} \rightarrow S^{1} * S^{1}$ da forma $T_{p, q}(\theta)=\left(p \theta, q \theta, \frac{1}{2}\right)$.

Definição 1.0.6. $T_{p, q}$ (ou sua imagem) em $S^{3}$ é chamado nó toral (pois está contido no toro padrão $T)$ do tipo $(p, q)$.

Observe que se fixarmos os geradores padrões de $T$ o nó $T_{p, q}$ dá $p$ voltas na direção longitudinal e $q$ voltas na direção do meridiano ("dependendo da situação do observador"). Por exemplo o nó trefoil é do tipo $(2,3)$.

É fácil calcular o grupo destes nós usando-se a decomposiçāo do seu complementar subjacente a decomposição de $S^{3}$ pelo toro padrão acima e o teorema de Van Kampen. Obtemos $G_{p, q}=\left|x, y: x^{p}=y^{q}\right|$, o grupo do nó $T_{p, q}$. 
Proposição 1.0.3. [20] Dado um nó $K^{n} \subset S^{n+2}$ existe uma variedade compacta, conexa e orientável $M^{n+1} \subset S^{n+2}$ tal.que $\partial M^{n+1}=K^{n}$.

Definição 1.0.7. Qualquer variedade da forma acima é chamada Variedade de Seifert para o nó $K$.

Veja exemplo abaixo:

(a) Para o nó trefoil abaixo a superfície desenhada (Faixa de Möbius) nāo é uma superfície de Seifert para o trefoil, pois não é orientável.

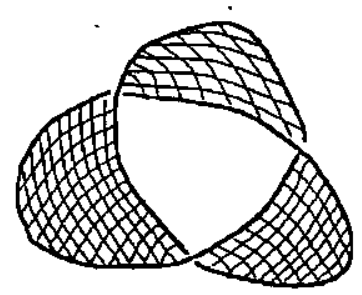

(b) O nó abaixo é o trefoil e a superfície que o borda (constituída por dois discos $D_{1}^{2}$ e $D_{2}^{2}$ colados por 3 retângulos torcidos) é orientável, e portanto é uma superfície de Seifert para o trefoil.

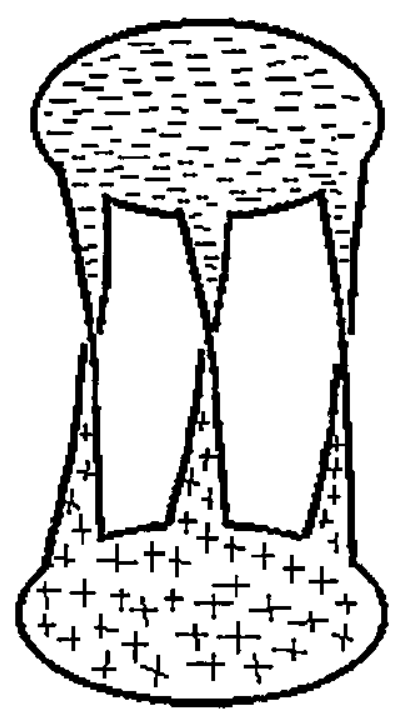


Definição 1.0.8. $O$ genus de um nó ou enlaçamento $K^{1} e m R^{3}$ ou $S^{3}$ é o menor genus de todas as superfícies Seifert do nó. Escreva $g\left(K^{1}\right)$ para este valor. Genus é um invariante do nó.

Observação 1.0.2. O genus de uma superfície com bordo é o genus de sua superfície associada (isto é, a superfície fechada obtida colando-se um disco em cada componente de bordo).

Teorema 1.0.1. (Teorema do Toro Sólido) [20] Todo toro $\left(S^{1} \times S^{1}\right)$ mergulhado em $S^{3}$, divide este espaço em dias componentes conexas e o fecho de pelo menos uma delas é um toro sólido $\left(S^{1} \times D^{2}\right)$.

Definição 1.0.9. (Espaços Lenticulares) Sejam dois toros sólidos $V_{1}$ e $V_{2}$ e um homeomorfismo $f: \partial V_{2} \rightarrow \partial V_{1}$. A colagem $V_{1} \cup_{h} V_{2}=M^{3}$ é uma variedade compacta, conexa, orientável sem bordo de dimensão 3, que é chamada Espaço Lenticular.

Observação 1.0.3. Fixando-se longitude e meridiano padrões $l_{1}$ e $m_{1}$ para $\partial V_{1}$, e $l_{2}$ e $m_{2}$ para $\partial V_{2}$, podemos escrever $f_{*}\left(m_{2}\right)=p l_{1}+q m_{1}$ onde $p$ e $q$ são inteiros coprimos. $O$ espaço lenticular obtido é denotado $L(p, q)$.

Em outras palavras, uma 3-variedade é um espaço lenticular se e somente se contém um toro sólido cujo fecho do complemento é também um toro sólido.

Alguns escritores não consideram $S^{3}$ e $S^{2} \times S^{1}$ como espaços lenticulares.

Definição 1.0.10. Seja $M^{m}$ uma subvariedade de dimensão $m$ de uma variedade $N^{n}$ de dimensão n. Então existe uma vizinhança de $M^{m}$ em $N^{n}$ que localmente 
é da forma $U \times D^{n-m}$, onde $U$ é aberto de $M^{m}$. Esta vizinhança é chamadr vizinhança tubular de $M^{m}$ em $N^{n}$.

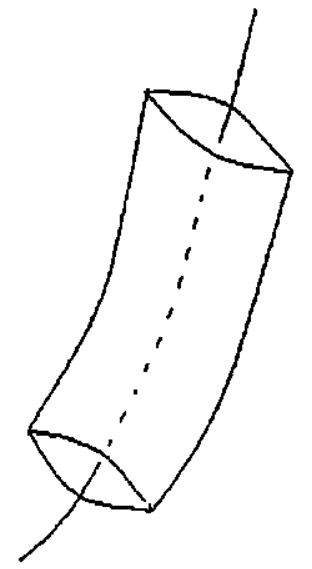

Quando o fibrado normal de $M^{m}$ em $N^{n}$ é trivial, então podemos obter uma vizinhança da forma $M^{m} \times D^{n-m}$.

Observaçāo 1.0.4. Nem sempre a vizinhança tubular de uma subvariedade $M \subset$ $N$ é trivial, isto é, nem sempre é da forma $M \times D^{k}$. Por exemplo na faixa de Möbius, se tomarmos uma vizinhança tubular do círculo central da faixa, obtemos uma outra faixa de Möbius, ao passo que se tomarmos um círculo (homotópico à constante) na faixa de Möbius, obteremos um cilindro.

Seja $M$ uma variedade com bordo $\partial M(\neq \emptyset)$. $\partial M$ é subvariedade, porém não possui vizinhança tubular, contudo possui uma "meia vizinhança tubular" chamada usualmente de colarinho.

Definiçāo 1.0.11. Dada uma variedade $M$ com bordo $\partial M$ um mergulho $f: \partial M \times$ $[0, \infty) \rightarrow M$ tal que $f(x, 0)=x$ é chamado um colarinho de $\partial M$ em $M$.

Proposiçāo 1.0.4. (Teorema do Colarinho) [14] Para toda variedade $M$ com bordo $\partial M$ existe um colarinho, e dois colarinhos são isotópicos. 
Definição 1.0.12. Seja $M^{3}$ uma variedade de dimensão 3 e seja $V$ um toro sólido em $M^{3}$, isto é, $V$ 'é a vizinhança tubular de um nó em $M^{3}$. Removendo o toro de $M^{3}$ e colando-o novamente por um homeomorfismo no bordo, obtemos uma outra variedade $N^{3}$. (Dependendo do homeomorfismo pode-se obter a mesma $\left.M^{3}\right)$. Esta operação é chamada de Dehn twist.

Definição 1.0.13. (Homeomorfismo Pseudo-Anosov) Assuma que $f: S \rightarrow$ $S$ seja um certo homeomorfismo, que preserva orientação, de uma superfície orientada, compacta e conexa. O homeomorfismo $f$ é redutível se $f(C)=C$ para alguma união não vazia $C$ de curvas fechadas simples disjuntas essenciais tal que duas curvas não são homotópicas e nenhuma curva é homotópica a uma componente de bordo de $S$. Chamamos $C$ uma 1-variedade redutora para $f$. Após uma isotopia podemos assumir que f fixa uma vizinhança $N(C)$ de $C$ consistindo de anéis fechados disjuntos. Cada componente $S_{i}$ de $S$-int $N(C)$ é invariante em relação à uma iteração mínima $f^{k_{i}}$. Chamamos $\left.f^{k_{i}}\right|_{S_{i}}: S_{i} \rightarrow S_{i}$ uma componente de $f$, e denotamos por $f_{i}$. Se $f$ não é redutivel, então olhamos $f_{1}(=f): S \rightarrow S$ como a única componente. Um homeomorfismo $f: S \rightarrow S$ de uma superfície $S$ compacta, conexa e orientável com característica de Euler negativa e possivelmente com bordo é pseudo-Anosov se existe um par transverso de folheações f-invariantes mensuráveis tendo um número finito de singularidades (com restrições a tipo de singularidades no interior de $S$ ) tal que $f$ expande unifomemente as folhas de uma folheação pelo fator $\lambda$ e a outra folheação pelo fator $\lambda^{-1}$. $O$ fator $\lambda$ está bem definido, e é chamado a dilatação de $f$.

Proposição 1.0.5. [23] Seja $f: S \rightarrow S$ um homeomorfismo que preserva orientação de uma superfície compacta, conexa e orientada com característica de Euler negativa e possivelmente com bordo. Após uma isotopia conveniente de $f$, uma das seguintes condições ocorre:

(i) f é periódica; 
(ii) $f$ é pseudo-Anosov;

(iii) $f$ é redutível, e cada componente de $f$ é periódica ou pseudo-Anosov. Além disso, sobre cada componente de $N(C)$ uma última iteração de $f$ que deixa componente invariante é um Dehn twist.

Observação 1.0.5. Se a 1-variedade redutora $C$ na Proposição anterior (iii) é mínima no sentido que ela não contém subvariedades próprias satisfazendo a conclusão da Proposição, então $C$ é única a menos de isotopia.

Definição 1.0.14. Seja $M$ uma 3-variedade e F uma superfície que está propriamente mergulhada em $M$ ou contida em $\partial M$. Diremos que $F$ é incompressivel em $M$ se nenhuma das seguintes condições ocorre:

(i) F é uma 2-esfera que borda uma 3-célula homotópica em $M$, ou

(ii) F é uma 2-célula e, ou $F \subset \dot{\partial} M$ ou existe uma 3-célula homotópica $X \subset M \operatorname{com} \partial X \subset F \cup \partial M$, ou

(iii) existe uma 2-célula $D \subset M$ com $D \cap F=\partial D$ e com $\partial D$ não contraível em $F$.

Definição 1.0.15. Um sistema dinâmico (clássico) é um par $\Sigma=(X, \sigma)$, onde $X$ é um espaço não vazio, compacto e Hausdorff, e $\sigma: X \rightarrow X$ é uma aplicação contínua. $\Sigma$ é um sistema inversível se $\sigma$ é inversível.

Definição 1.0.16. Um sistema dinâmico $\Sigma=(X, \sigma)$ é equicontínuo se a coleção $\left\{\sigma^{n}: n \in \mathbb{Z}\right\}$ de transformações de $X$ é equicontínua.

Proposição 1.0.6. (Teorema de Tychonoff): Um produto arbitrário de espaços compactos é compacto na topologia produto. 
Proposição 1.0.7. [9] Seja $\left(a_{n}\right)_{n \geq 1} e\left(b_{n}\right)_{n \geq 1}$ duas sequências com $a_{n}, b_{n} \geq 0 e$ $k>0$. Temos:

(i) $\lim \sup \frac{1}{n} \log \left(a_{n}+b_{n}\right)=\max \left(\lim \sup \frac{1}{n} \log a_{n}, \lim \sup \frac{1}{n} \log b_{n}\right)$;

(ii) $\limsup \frac{1}{n} \log k a_{n}=\lim \sup \frac{1}{n} \log a_{n}$;

(iii) $\lim \sup \frac{1}{n} \log a_{n} \leq \lim \sup \frac{1}{n} \log \left(a_{1}+\ldots+a_{n}\right) \leq \max \left(0, \lim \sup \frac{1}{n} \log a_{n}\right)$. 


\section{Capítulo 2}

\section{Entropia Topológica}

O conteúdo deste capítulo teve como referência básica o artigo [1]. Foram detalhadas algumas demonstraçōes e feitos alguns exemplos. A proposta deste capítulo é introduzir a noção de entropia como um invariante para aplicações contínuas.

\subsection{Definições e Propriedades Gerais}

Seja $X$ um espaço topológico compacto.

Definição 2.1.1. Para qualquer cobertura aberta $\mathfrak{U}$ de $X$, seja $N(\mathfrak{U})$ o número de conjuntos em uma subcobertura de cardinalidade mínima. Uma subcobertura de uma cobertura é mínima se nenhuma outra contém menos membros. Como $X$ é compacto e $\mathfrak{U}$ é uma cobertura aberta, existe sempre uma subcobertura finita. Chamamos $H(\mathfrak{U})=\log N(\mathfrak{U})$ a entropia de $\mathfrak{U}$.

Definiçāo 2.1.2. Para quaisquer duas coberturas $\mathfrak{U}, \mathfrak{B}$, a cobertura $\mathfrak{U} \vee \mathfrak{B} \equiv$ $\{A \cap B \mid A \in \mathfrak{U}, B \in \mathfrak{B}\}$ chama-se join de $\mathfrak{U}$ e $\mathfrak{B}$. 
Definição 2.1.3. Uma cobertura $\mathfrak{B}$ é dita um refinamento de uma cobertura $\mathfrak{L}$, e denotamos $\mathfrak{U} \prec \mathfrak{B}$, se todo membro de $\mathfrak{B}$ é um subconjunto de algum membro de $\mathfrak{U}$.

Temos as seguintes propriedades básicas.

Propriedade 2.1.1. A operação $\vee$ é comutativa e associativa.

\section{Demonstraçāo:}

Comutativa: Sejam $\mathfrak{U}, \mathfrak{B}$ duas coberturas de $X$. Temos que:

$\mathfrak{U} \vee \mathfrak{B} \equiv\{A \cap B \mid A \in \mathfrak{U}, B \in \mathfrak{B}\} \equiv\{B \cap A \mid B \in \mathfrak{B}, A \in \mathfrak{U}\} \equiv \mathfrak{B} \vee \mathfrak{U}$ Portanto, $\mathfrak{U} \vee \mathfrak{B} \equiv \mathfrak{B} \vee \mathfrak{U}$.

Associativa: Sejam $\mathfrak{U}, \mathfrak{B}, \mathfrak{C}$ coberturas de $X$. Temos que: $(\mathfrak{U} \vee \mathfrak{B}) \vee \mathfrak{C} \equiv\{(A \cap B) \mid A \in \mathfrak{U}$ e $B \in \mathfrak{B}\} \vee \mathfrak{C} \equiv\{(A \cap B) \cap C \mid(A \cap B) \in \mathfrak{U} \vee \mathfrak{B}$ e $C \in \mathfrak{C}\} \equiv\{(A \cap B) \cap C \mid A \in \mathfrak{U}, B \in \mathfrak{B}$ e $C \in \mathfrak{C}\} \equiv\{A \cap(B \cap C) \mid A \in \mathfrak{U}, B \in \mathfrak{B}$ e $C \in \mathfrak{C}\} \equiv\{A \cap(B \cap C) \mid A \in \mathfrak{U}$ e $(B \cap C) \in \mathfrak{B} \vee \mathfrak{C}\} \equiv \mathfrak{U} \vee(\mathfrak{B} \vee \mathfrak{C})$

Portanto, $(\mathfrak{U} \vee \mathfrak{B}) \vee \mathfrak{C} \equiv \mathfrak{U} \vee(\mathfrak{B} \vee \mathfrak{C})$.

Propriedade 2.1.2. A relação $\prec$ é uma ordem parcial reflexiva sobre a familia de coberturas abertas de $X$.

Demonstração: Seja $\mathfrak{B}$ uma cobertura aberta de $X$, temos que $\mathfrak{B} \prec \mathfrak{B}$, logo vale a propriedade reflexiva.

Sejam agora $\mathfrak{B}, \mathfrak{U}, \mathfrak{C}$ coberturas abertas de $X$ tal que $\mathfrak{U} \prec \mathfrak{B}$ e $\mathfrak{C} \prec \mathfrak{U}$.

$\mathfrak{U} \prec \mathfrak{B} \Rightarrow \forall B \in \mathfrak{B}, \exists A \in \mathfrak{U} \mid B \subset A$

$\mathfrak{C} \prec \mathfrak{U} \Rightarrow \forall A \in \mathfrak{U}, \exists C \in \mathfrak{C} \mid A \subset C$.

Assim $\forall B \in \mathfrak{B}, \exists C \in \mathfrak{C} \mid B \subset C$. Logo $\mathfrak{C} \prec \mathfrak{B}$.

Portanto, a relação $\prec$ é uma ordem parcial reflexiva. 
Propriedade 2.1.3. $\mathfrak{U} \prec \mathfrak{U}^{\prime}, \mathfrak{B} \prec \mathfrak{B}^{\prime} \Rightarrow \mathfrak{U} \vee \mathfrak{B} \prec \mathfrak{U}^{\prime} \vee \mathfrak{B}^{\prime}$

Demonstração: Considere $A^{\prime} \cap B^{\prime} \in \mathfrak{U}^{\prime} \vee \mathfrak{B}^{\prime}$, onde $A^{\prime} \in \mathfrak{U}^{\prime}$ e $B^{\prime} \in \mathfrak{B}^{\prime}$. Por hipótese temos que $\mathfrak{U} \prec \mathfrak{U}^{\prime}$ e $\mathfrak{B} \prec \mathfrak{B}^{\prime}$, assim existe $A \in \mathfrak{U}$ e $B \in \mathfrak{B}$ tal que $A^{\prime} \subset A, B^{\prime} \subseteq B$.

Assim $A^{\prime} \cap B^{\prime} \subseteq A \cap B$ onde $A \cap B \in \mathfrak{U} \vee \mathfrak{B}$.

Portanto $\mathfrak{U} \vee \mathfrak{B} \prec \mathfrak{U}^{\prime} \vee \mathfrak{B}^{\prime}$.

Observação 2.1.1. Com as próprias substituições de $\mathfrak{U}, \mathfrak{B}$ e a cobertura $\mathfrak{X}$ no enunciado acima (Propriedade 2.1.3) obtemos $\mathfrak{U} \prec \mathfrak{U} \vee \mathfrak{B}$ e $\mathfrak{B} \prec \mathfrak{U} \vee \mathfrak{B}$ que revela que a familia de coberturas abertas é um conjunto ordenado com respeito à relação $\prec$.

$\mathfrak{U} \prec \mathfrak{U}, \mathfrak{U} \prec \mathfrak{B} \Rightarrow \mathfrak{U} \prec \mathfrak{U} \vee \mathfrak{B}$.

$\mathfrak{B} \prec \mathfrak{B}, \mathfrak{B} \prec \mathfrak{U} \Rightarrow \mathfrak{B} \prec \mathfrak{U} \vee \mathfrak{B}$.

Propriedade 2.1.4. $\mathfrak{U} \prec \mathfrak{B} \Rightarrow N(\mathfrak{U}) \leq N(\mathfrak{B}), H(\mathfrak{l}) \leq H(\mathfrak{B})$.

Demonstração: Seja $\left\{B_{1}, \ldots, B_{N(\mathfrak{B})}\right\}$ uma subcobertura mínima de $\mathfrak{B}$. Como $\mathfrak{U} \prec \mathfrak{B}$, existe uma subcobertura $\left\{A_{1}, \ldots, A_{N(\mathfrak{B})}\right\}$ de $\mathfrak{U}$, tal que

$$
\begin{aligned}
& B_{1} \subset A_{1}, \\
& B_{2} \subset A_{2}, \\
& \quad \vdots \\
& B_{N(\mathfrak{B})} \subset A_{N(\mathfrak{B})} . \text { Logo } \\
& \cup B_{i} \subset \cup A_{i}, i=1,2, \ldots, N(\mathfrak{B})
\end{aligned}
$$

e como $\cup B_{i}$ cobre $X$ temos que $\cup A_{i}$ cobre $X$. Mas $\left\{A_{1}, \ldots, A_{N(\mathfrak{B})}\right\}$ não necessariamente é mínima.

Portanto $N(\mathfrak{U}) \leq N(\mathfrak{B})$.

Como $\log$ é função.crescente, $\log N(\mathfrak{l}) \leq \log N(\mathfrak{B})$ e assim temos que $H(\mathfrak{U}) \leq H(\mathfrak{B})$. 
Propriedade 2.1.5. $\mathfrak{U} \prec \mathfrak{B} \Rightarrow N(\mathfrak{U} \vee \mathfrak{B})=N(\mathfrak{B})$ e $H(\mathfrak{U} \vee \mathfrak{B})=H(\mathfrak{B})$.

Demonstração: Pela Propriedade 2.1.3, temos que $\mathfrak{B} \prec \mathfrak{U} \vee \mathfrak{B}$ e assim pela Propriedade 2.1.4 temos que $N(\mathfrak{B}) \leq N(\mathfrak{U} \vee \mathfrak{B})$.

Por outro lado, por hipótese $\mathfrak{U} \prec \mathfrak{B}$ e sabemos que $\mathfrak{B} \prec \mathfrak{B}$, assim $\mathfrak{U} \vee \mathfrak{B} \prec \mathfrak{B}$.

Portanto, novamente, pela Propriedade 2.1.4, temos que $N(\mathfrak{U} \vee \mathfrak{B}) \leq N(\mathfrak{B})$.

Logo $N(\mathfrak{U} \vee \mathfrak{B})=N(\mathfrak{B})$.

Como $H(\mathfrak{U} \vee \mathfrak{B})=\log N(\mathfrak{U} \vee \mathfrak{B})$ e $H(\mathfrak{B})=\log N(\mathfrak{B})$, temos

$$
H(\mathfrak{U} \vee \mathfrak{B})=H(\mathfrak{B})
$$

Propriedade 2.1.6. $N(\mathfrak{U} \vee \mathfrak{B}) \leq N(\mathfrak{U}) . N(\mathfrak{B})$ e $H(\mathfrak{U} \vee \mathfrak{B}) \leq H(\mathfrak{U})+H(\mathfrak{B})$.

Demonstração: Sejam $\left\{A_{1}, \ldots, A_{N(\mathfrak{U})}\right\}$ uma subcobertura de $\mathfrak{U}$ e $\left\{B_{1}, \ldots, B_{N(\mathfrak{B})}\right\}$ uma subcobertura de $\mathfrak{B}$. Então $\left\{A_{i} \cap B_{j} \mid i=1, \ldots, N(\mathfrak{U}), j=1, \ldots, N(\mathfrak{B})\right\}$ é uma subcobertura de $\mathfrak{U} \vee \mathfrak{B}$.

Consequentemente $N(\mathfrak{U} \vee \mathfrak{B}) \leq N(\mathfrak{U}) \cdot N(\mathfrak{B})$.

Como log é função crescente temos que $\log N(\mathfrak{U} \vee \mathfrak{B}) \leq \log [N(\mathfrak{U}) \cdot N(\mathfrak{B})]=\log N(\mathfrak{U})+\log N(\mathfrak{B})$ $\Rightarrow \log N(\mathfrak{U} \vee \mathfrak{B}) \leq \log N(\mathfrak{U})+\log N(\mathfrak{B})$.

Portanto, $H(\mathfrak{U} \vee \mathfrak{B}) \leq H(\mathfrak{U})+H(\mathfrak{B})$.

Seja $\varphi: X \rightarrow X$ uma aplicação contínua. Seja $\mathfrak{U}$ uma cobertura aberta de $X$ então da continuidade de $\varphi$, a família de $\varphi^{-1} \mathfrak{U}=\left\{\varphi^{-1} A \mid A \in \mathfrak{U}\right\}$ é novamente uma cobertura aberta. 
Propriedade 2.1.7. $\mathfrak{U} \prec \mathfrak{B} \Rightarrow \varphi^{-1} \mathfrak{U} \prec \varphi^{-1} \mathfrak{B}$.

Demonstração: Temos que:

$\varphi^{-1} \mathfrak{U}=\left\{\varphi^{-1} A \mid A \in \mathfrak{U}\right\}$,

$\varphi^{-1} \mathfrak{B}=\left\{\varphi^{-1} B \mid B \in \mathfrak{B}\right\}$.

Seja $\varphi^{-1} B \in \varphi^{-1} \mathfrak{B}$. Como $\mathfrak{U} \prec \mathfrak{B} \Rightarrow$ dado $B \in \mathfrak{B}, \exists A \in \mathfrak{U}$ tal que $B \subset A \Rightarrow$ $\varphi^{-1} B \subset \varphi^{-1} A$, onde $\varphi^{-1} A \in \varphi^{-1} \mathfrak{U}$.

Portanto, $\varphi^{-1} \mathfrak{U} \prec \varphi^{-1} \mathfrak{B}$.

Propriedade 2.1.8. $\varphi^{-1}(\mathfrak{U} \vee \mathfrak{B})=\varphi^{-1} \mathfrak{U} \vee \varphi^{-1} \mathfrak{B}$

Demonstração: Temos que:

$\mathfrak{U} \vee \mathfrak{B}=\{A \cap B \mid A \in \mathfrak{U}, B \in \mathfrak{B}\}$,

$\varphi^{-1}(\mathfrak{U} \vee \mathfrak{B})=\left\{\varphi^{-1}(A \cap B)\right.$, onde $\left.A \cap B \in \mathfrak{U} \vee \mathfrak{B}\right\}$ e

$\varphi^{-1} \mathfrak{U}=\left\{\varphi^{-1} A \mid A \in \mathfrak{U}\right\}$ e $\varphi^{-1} \mathfrak{B}=\left\{\varphi^{-1} B \mid B \in \mathfrak{B}\right\}$.

Assim,

$$
\begin{aligned}
\varphi^{-1} \mathfrak{U} \vee \varphi^{-1} \mathfrak{B} & =\left\{\varphi^{-1} A \cap \varphi^{-1} B \mid \varphi^{-1} A \in \varphi^{-1} \mathfrak{U} \text { e } \varphi^{-1} B \in \varphi^{-1} \mathfrak{B}\right\} \\
& =\left\{\varphi^{-1}(A \cap B) \mid A \cap B \in \mathfrak{U} \vee \mathfrak{B}\right\}=\varphi^{-1}(\mathfrak{U} \vee \mathfrak{B}) .
\end{aligned}
$$

Portanto $\varphi^{-1} \mathfrak{U} \vee \varphi^{-1} \mathfrak{B}=\varphi^{-1}(\mathfrak{U} \vee \mathfrak{B})$.

Propriedade 2.1.9. $N(\mathfrak{U}) \geq N\left(\varphi^{-1} \mathfrak{U}\right)$.

Demonstração: Seja $\left\{A_{1}, \ldots, A_{N(\mathfrak{l})}\right\}$ uma subcobertura mínima de $\mathfrak{U}$. Como $\left\{\varphi^{-1} A_{1}, \ldots, \varphi^{-1} A_{N(\mathfrak{l})}\right\}$ é uma cobertura, possivelmente não mínima, temos $N\left(\varphi^{-1} \mathfrak{U}\right) \leq N(\mathfrak{U})$.

Observação 2.1.2. Quando $\varphi$ é sobrejetora então $N(\mathfrak{U})=N\left(\varphi^{-1} \mathfrak{U}\right)$, pois neste caso a cobertura $\left\{\varphi^{-1} A_{1}, \ldots, \varphi^{-1} A_{N(\varkappa)}\right\}$ é mínima ja que se fosse possivel eliminar um de seus elementos a sua imagem também poderia ser eliminada de $\left\{A_{1}, \ldots, A_{N(\mathfrak{l})}\right\}$. 
Propriedade 2.1.10.

$$
\lim _{n \rightarrow \infty} \frac{H\left(\bigvee_{k=0}^{n-1} \varphi^{-k} \mathfrak{U}\right)}{n}=\lim _{n \rightarrow \infty} \frac{H\left(\mathfrak{U} \vee \varphi^{-1} \mathfrak{U} \vee \ldots \vee \varphi^{-n+1} \mathfrak{U}\right)}{n}
$$

existe e é finito.

Demonstração:

$$
\begin{aligned}
H\left(\mathfrak{U} \vee \ldots \vee \varphi^{-m-n+1} \mathfrak{U}\right) & =H\left(\mathfrak{U} \vee \ldots \vee \varphi^{-m+1} \mathfrak{U} \vee \varphi^{-m} \mathfrak{U} \vee \varphi^{-m-1} \mathfrak{U} \vee \ldots \vee \varphi^{-m-n+1} \mathfrak{U}\right) \\
& =H\left(\mathfrak{U} \vee \ldots \vee \varphi^{-m+1} \mathfrak{U} \vee \varphi^{-m}\left(\mathfrak{U} \vee \varphi^{-1} \mathfrak{U} \vee \ldots \vee \varphi^{-n+1} \mathfrak{U}\right)\right) \\
& \leq H\left(\mathfrak{U} \vee \ldots \vee \varphi^{-m+1} \mathfrak{U}\right)+H\left(\varphi^{-m}\left(\mathfrak{U} \vee \ldots \vee \varphi^{-n+1} \mathfrak{U}\right)\right) \\
& \leq H\left(\mathfrak{U} \vee \ldots \vee \varphi^{-m+1} \mathfrak{U}\right)+H\left(\mathfrak{U} \vee \ldots \vee \varphi^{-n+1} \mathfrak{U}\right) .
\end{aligned}
$$

A segunda igualdade segue da Propriedade 2.1.8; a próxima desigualdade da Propriedade 2.1.6; e a desigualdade final da Propriedade 2.1.9.

Portanto, $H\left(\mathfrak{U} \vee \ldots \vee \varphi^{-m-n+1} \mathfrak{U}\right) \leq H\left(\mathfrak{U} \vee \ldots \vee \varphi^{-m+1} \mathfrak{U}\right)+H\left(\mathfrak{U} \vee \ldots \vee \varphi^{-n+1} \mathfrak{U}\right)$

Seja $H_{n}=H\left(\mathfrak{U} \vee \ldots \vee \varphi^{-n+1} \mathfrak{U}\right)$. Assim temos $H_{m+n} \leq H_{m}+H_{n}$ e $H_{n} \geq 0$, para todo inteiro positivo $m, n$.

Basta mostrar que

$$
\lim _{n \rightarrow \infty} \frac{H_{n}}{n}
$$

existe e é finito.

Observemos que $H_{k m} \leq k H_{m}$ pois, por hipótese sabemos que $H_{m+n} \leq H_{m}+H_{n}$, para qualquer $m, n$. Em particular quando $n=m$. Assim,

$$
\begin{aligned}
& H_{m+m}=H_{2 m} \leq H_{m}+H_{m}=2 H_{m} \Rightarrow H_{2 m} \leq 2 H_{m}, \\
& H_{2 m+m}=H_{3 m} \leq H_{2 m}+H_{m} \leq 2 H_{m}+H_{m}=3 H_{m} \Rightarrow H_{3 m} \leq 3 H_{m}, \\
& \quad \vdots \\
& H_{(k-1) m+m}=H_{k m} \leq H_{(k-1) m}+H_{m} \leq(k-1) H_{m}+H_{m}=k H_{m} \Rightarrow H_{k m} \leq k H_{m} .
\end{aligned}
$$


Fixe $m>0$. Para cada $j>0$ seja $j=k m+n$ onde $0 \leq n<m$.

Então

$$
\frac{H_{j}}{j}=\frac{H_{n+k m}}{n+k m} \leq \frac{H_{n}}{k m}+\frac{H_{k m}}{k m} \leq \frac{H_{n}}{k m}+\frac{k H_{m}}{k m}=\frac{H_{n}}{k m}+\frac{H_{m}}{m} .
$$

Portanto,

$$
\frac{H_{j}}{j} \leq \frac{H_{n}}{k m}+\frac{H_{m}}{m}
$$

Temos que $m$ está fixo e $n$ varia entre 0 e $m$, e quando $j \rightarrow \infty$ temos $k \rightarrow \infty$. Assim

$$
\lim _{j \rightarrow \infty} \sup \frac{H_{j}}{j} \leq \lim _{j \rightarrow \infty} \sup \left(\frac{H_{n}}{k m}+\frac{H_{m}}{m}\right)=\lim _{k \rightarrow \infty} \sup \left(\frac{H_{n}}{k m}+\frac{H_{m}}{m}\right)=\frac{H_{m}}{m} .
$$

Portanto, $\lim _{j \rightarrow \infty} \sup \frac{H_{j}}{j} \leq \frac{H_{m}}{m}$ e daí $\lim _{j \rightarrow \infty} \sup \frac{H_{j}}{j} \leq \inf \frac{H_{m}}{m}$, pois se é menor ou igual que qualquer $\frac{H_{m}}{m}$ é menor ou igual ao inf.

Por definição liminf $H_{n}=\sup _{n} \inf _{k \geq n} H_{k}$. Logo, inf $\frac{H_{m}}{m} \leq \lim _{j \rightarrow \infty} \inf \frac{H_{j}}{j}$ já que do lado esquerdo da desigualdade temos o inf e do lado direito o sup dos inf.

Assim temos que:

$$
\lim _{j \rightarrow \infty} \sup \frac{H_{j}}{j} \leq \inf \frac{H_{m}}{m} \leq \lim _{j \rightarrow \infty} \inf \frac{H_{j}}{j} \Rightarrow \lim _{j \rightarrow \infty} \sup \frac{H_{j}}{j} \leq \lim _{j \rightarrow \infty} \inf \frac{H_{j}}{j} .
$$

Além disso, sabemos que $\lim _{j \rightarrow \infty} \inf \frac{H_{j}}{j} \leq \lim _{j \rightarrow \infty} \sup \frac{H_{j}}{j}$. Assim temos que $\lim _{j \rightarrow \infty} \inf \frac{H_{j}}{j}=\lim _{j \rightarrow \infty} \sup \frac{H_{j}}{j}$. Portanto temos, $\lim _{j \rightarrow \infty} \inf \frac{H_{j}}{j}=\inf \frac{H_{m}}{m}=\lim _{j \rightarrow \infty} \sup \frac{H_{j}}{j}$.

Assim concluímos que $\lim _{j \rightarrow \infty} \frac{H_{j}}{j}$ existe e é igual a inf $\frac{H_{m}}{m}$. 
Definição 2.1.4. A entropia $h(\varphi, \mathfrak{U})$ de uma aplicação $\varphi$ com respeito a uma cobertura $\mathfrak{U}$ é definida como

$$
h(\varphi, \mathfrak{U})=\lim _{n \rightarrow \infty} \frac{H\left(\mathfrak{U} \vee \varphi^{-1} \mathfrak{U} \vee \ldots \vee \varphi^{-n+1} \mathfrak{U}\right)}{n}
$$

Propriedade 2.1.11. $h(\varphi, \mathfrak{U}) \leq H(\mathfrak{U})$.

Demonstração: Temos que:

$$
\begin{gathered}
h(\varphi, \mathfrak{U})=\lim _{n \rightarrow \infty} \frac{H\left(\mathfrak{U} \vee \varphi^{-1} \mathfrak{U} \vee \ldots \vee \varphi^{-n+1} \mathfrak{U}\right)}{n}=\lim _{n \rightarrow \infty} \frac{\log N\left(\mathfrak{U} \vee \varphi^{-1} \mathfrak{U} \vee \ldots \vee \varphi^{-n+1} \mathfrak{U}\right)}{n} \\
\leq \lim _{n \rightarrow \infty} \frac{1}{n}\left[\log \left[N(\mathfrak{U}) \cdot N\left(\varphi^{-1} \mathfrak{U}\right) \ldots N\left(\varphi^{-n+1} \mathfrak{U}\right)\right]\right] \leq \lim _{n \rightarrow \infty} \frac{1}{n}[\log [N(\mathfrak{U}) . N(\mathfrak{U}) \ldots N(\mathfrak{U})]] \\
\leq \lim _{n \rightarrow \infty} \frac{1}{n}\left[\log N(\mathfrak{U})^{n}\right]=\lim _{n \rightarrow \infty} \frac{1}{n}[n \log N(\mathfrak{U})] \\
=\lim _{n \rightarrow \infty} \log N(\mathfrak{U})=\log N(\mathfrak{U})=H(\mathfrak{U}) .
\end{gathered}
$$

Portanto,

$$
h(\varphi, \mathfrak{U}) \leq H(\mathfrak{U})
$$

Observemos que a primeira desigualdade segue da Propriedade 2.1.6 e a segunda desigualdade segue da Propriedade 2.1.9. 
Propriedade 2.1.12. $\mathfrak{U} \prec \mathfrak{B} \Rightarrow h(\varphi, \mathfrak{U}) \leq h(\varphi, \mathfrak{B})$.

Demonstraçāo: Como por hipótese $\mathfrak{U} \prec \mathfrak{B}$ temos pela Propriedade 2.1.7 que $\varphi^{-1} \mathfrak{U} \prec \varphi^{-1} \mathfrak{B}$. Assim pela Propriedade 2.1.3 temos que $\mathfrak{U} \vee \varphi^{-1} \mathfrak{U} \prec \mathfrak{B} \vee \varphi^{-1} \mathfrak{B}$.

Podemos também generalizar

$$
\mathfrak{U} \vee \varphi^{-1} \mathfrak{U} \vee \ldots \vee \varphi^{-n+1} \mathfrak{U} \prec \mathfrak{B} \vee \varphi^{-1} \mathfrak{B} \vee \ldots \vee \varphi^{-n+1} \mathfrak{B}
$$

Pela Propriedade 2.1.4, temos que:

$$
H\left(\mathfrak{U} \vee \varphi^{-1} \mathfrak{U} \vee \ldots \vee \varphi^{-n+1} \mathfrak{U}\right) \leq H\left(\mathfrak{B} \vee \varphi^{-1} \mathfrak{B} \vee \ldots \vee \varphi^{-n+1} \mathfrak{B}\right)
$$

Logo

$$
\begin{gathered}
\lim _{n \rightarrow \infty} H\left(\mathfrak{U} \vee \varphi^{-1} \mathfrak{U} \vee \ldots \vee \varphi^{-n+1} \mathfrak{U}\right) \leq \lim _{n \rightarrow \infty} H\left(\mathfrak{B} \vee \varphi^{-1} \mathfrak{B} \vee \ldots \vee \varphi^{-\mathfrak{n}+\mathfrak{l}} \mathfrak{B}\right) \\
\Rightarrow h(\varphi, \mathfrak{U}) \leq h(\varphi, \mathfrak{B}) .
\end{gathered}
$$

Propriedade 2.1.13. Se $\varphi$ é um homeomorfismo, então $h(\varphi, \mathfrak{U})=h\left(\varphi^{-1}, \mathfrak{U}\right)$.

Demonstração: Temos que:

$$
\begin{aligned}
& \begin{aligned}
H\left(\mathfrak{U} \vee \ldots \vee \varphi^{-n+1} \mathfrak{U}\right) & =H\left(\varphi^{n-1}\left(\mathfrak{U} \vee \ldots \vee \varphi^{-n+1} \mathfrak{U}\right)\right) \\
& =H\left(\varphi^{n-1} \mathfrak{U} \vee \ldots \vee \varphi^{-n+1+n-1} \mathfrak{U}\right) \\
& =H\left(\mathfrak{U} \vee \varphi \mathfrak{U} \vee \ldots \vee \varphi^{n-1} \mathfrak{U}\right) \\
& =H\left(\mathfrak{U} \vee\left(\varphi^{-1}\right)^{-1} \mathfrak{U} \vee \ldots \vee\left(\varphi^{-1}\right)^{-n+1} \mathfrak{U}\right) .
\end{aligned} \\
& \Rightarrow H\left(\mathfrak{U} \vee \ldots \vee \varphi^{-n+1} \mathfrak{U}\right)=H\left(\mathfrak{U} \vee\left(\varphi^{-1}\right)^{-1} \mathfrak{U} \vee \ldots \vee\left(\varphi^{-1}\right)^{-n+1} \mathfrak{U}\right) . \\
& \text { Assim, } \\
& \qquad \lim _{n \rightarrow \infty} \frac{H\left(\mathfrak{U} \vee \ldots \vee \varphi^{-n+1} \mathfrak{U}\right)}{n}=\lim _{n \rightarrow \infty} \frac{H\left(\mathfrak{U} \vee\left(\varphi^{-1}\right)^{-1} \mathfrak{U} \vee \ldots \vee\left(\varphi^{-1}\right)^{-n+1} \mathfrak{U}\right)}{n} \\
& \quad \Rightarrow h(\varphi, \mathfrak{U})=h\left(\varphi^{-1}, \mathfrak{U}\right),
\end{aligned}
$$

onde a primeira igualdade segue da Propriedade 2.1.9. 
Definição 2.1.5. A entropia $h(\varphi)$ de uma aplicação $\varphi$ é definida como o $\sup h(\varphi, \mathfrak{U})$, onde o supremo é tomado sobre toda cobertura aberta $\mathfrak{U}$.

Definiçāo 2.1.6. Uma sequência $\left\{\mathfrak{U}_{n} \mid n=1,2, \ldots\right\}$ de coberturas abertas é refinada se

(1) $\mathfrak{U}_{n} \prec \mathfrak{U}_{n+1}$,

(2) Para toda cobertura aberta $\mathfrak{B}$ existe $\mathfrak{U}_{n}$ tal que $\mathfrak{B} \prec \mathfrak{U}_{n}$.

Uma sequência refinada de coberturas, quando existe, simplifica o cálculo de entropia como a próxima Propriedade revela.

Propriedade 2.1.14. Se $\left\{\mathfrak{U}_{n}\right\}$ é uma sequência refinada de coberturas,

$$
h(\varphi)=\lim _{n \rightarrow \infty} h\left(\varphi, \mathfrak{U}_{n}\right)
$$

Demonstração: Como $\left\{\mathfrak{U}_{n}\right\}$ é uma sequência refinada, temos que

$$
\mathfrak{U}_{n} \prec \mathfrak{U}_{n+1} \text { e } \forall \mathfrak{B}, \exists \mathfrak{U}_{n} \mid \mathfrak{B} \prec \mathfrak{U}_{n} .
$$

Assim, pela Propriedade 2.1.12, temos que

$$
h\left(\varphi, \mathfrak{U}_{n}\right) \leq h\left(\varphi, \mathfrak{U}_{n+1}\right) \text { e } h(\varphi, \mathfrak{B}) \leq h\left(\varphi, \mathfrak{U}_{n}\right), \forall \mathfrak{B} .
$$

Então $h(\varphi, \mathfrak{B}) \leq h\left(\varphi, \mathfrak{U}_{n}\right) \leq h\left(\varphi, \mathfrak{U}_{n+1}\right)$.

Portanto $\sup h\left(\varphi, \mathfrak{U}_{n}\right)=\lim _{n \rightarrow \infty} h\left(\varphi, \mathfrak{U}_{n}\right)$.

\subsection{Teoremas Gerais}

Teorema 2.2.1. Entropia é um invariante por conjugação, ou seja, $h\left(\psi \varphi \psi^{-1}\right)=$ $h(\varphi)$, onde

$\varphi: X \rightarrow X$ é contínua,

$\psi: X \rightarrow X^{\prime}$ é um homeomorfismo de $X$ para algum $X^{\prime}$. 
Demonstração: Seja $\mathfrak{U}$ cobertura aberta de X. Como $\psi$ é um homeomorfismo temos que $\psi \mathfrak{U}$ é cobertura aberta de $X^{\prime}$. Além disso,

$\mathfrak{U} \vee \varphi^{-1} \mathfrak{U} \vee \ldots \vee \varphi^{-n+1} \mathfrak{U}=\psi \mathfrak{U} \vee\left(\psi \varphi^{-1} \psi^{-1}\right) \psi \mathfrak{U} \vee \ldots \vee\left(\psi \varphi^{-n+1} \psi^{-1}\right) \psi \mathfrak{U}$

De fato:

Seja $\left\{A_{1}, A_{2}, \ldots, A_{n}\right\} \in \mathfrak{U} \vee \varphi^{-1} \mathfrak{U} \vee \ldots \vee \varphi^{-n+1} \mathfrak{U}$, onde $A_{1} \cup A_{2} \cup \ldots \cup A_{n}=X$.

Temos que $A_{i}=R \cap S \cap \ldots \cap T$, onde $R \in \mathfrak{U}, S \in \varphi^{-1} \mathfrak{U}, \ldots, T \in \varphi^{-n+1} \mathfrak{U}$.

Como $\varphi$ é um homeomorfismo temos que

$\psi A_{1} \cup \psi A_{2} \cup \ldots \cup \psi A_{n}=X^{\prime}$ e $\psi A_{i}=\psi(R) \cap \psi(S) \cap \ldots \cap \psi(T)$,

onde $\psi(R) \in \psi(\mathfrak{U}), \psi(S) \in\left(\psi \varphi^{-1} \psi^{-1}\right) \psi \mathfrak{U}, \ldots, \psi(T) \in\left(\psi \varphi^{-n+1} \psi^{-1}\right) \psi \mathfrak{U}$.

Assim temos que,

$h\left(\psi \varphi \psi^{-1}, \psi \mathfrak{U}\right)=\lim _{n \rightarrow \infty} \frac{H\left(\psi \mathfrak{U} \vee\left(\psi \varphi \psi^{-1}\right)^{-1} \psi \mathfrak{U} \vee \ldots \vee\left(\psi \varphi \psi^{-1}\right)^{-n+1} \psi \mathfrak{U}\right)}{n}$

$$
\begin{gathered}
\stackrel{(*)}{=} \lim _{n \rightarrow \infty} \frac{H\left(\psi \mathfrak{U} \vee\left(\psi \varphi^{-1} \psi^{-1}\right) \psi \mathfrak{U} \vee \ldots \vee\left(\psi \varphi^{-n+1} \psi^{-1}\right) \psi \mathfrak{U}\right.}{n} \\
=\lim _{n \rightarrow \infty} \frac{H\left(\mathfrak{U} \vee \varphi^{-1} \mathfrak{U} \vee \ldots \vee \varphi^{-n+1} \mathfrak{U}\right)}{n}=h(\varphi, \mathfrak{U}) .
\end{gathered}
$$

Portanto, $h\left(\psi \varphi \psi^{-1}, \dot{\psi} \mathfrak{U}\right)=h(\varphi, \mathfrak{U})$, para uma cobertura genérica.

Logo $h\left(\psi \varphi \psi^{-1}\right)=h(\varphi)$.

$\left(^{*}\right)\left(\psi \varphi \psi^{-1}\right)^{k}=\left(\psi \varphi \psi^{-1}\right)\left(\psi \varphi \psi^{-1}\right) \ldots\left(\psi \varphi \psi^{-1}\right)=\psi \varphi^{k} \psi^{-1}$.

Teorema 2.2.2. $h\left(\varphi^{k}\right)=k h(\varphi)$, para todo $k$, inteiro positivo.

Demonstração: Temos por definição que:

$h(\varphi, \mathfrak{U})=\lim _{n \rightarrow \infty} \frac{H\left(\mathfrak{U} \vee \varphi^{-1} \mathfrak{U} \vee \ldots \vee \varphi^{-n+1} \mathfrak{U}\right)}{n}$ e $h(\varphi)=\sup h(\varphi, \mathfrak{U})$.

Assim,

$$
h\left(\varphi^{k}\right) \geq h\left(\varphi^{k}, \mathfrak{U} \vee \varphi^{-1} \mathfrak{U} \vee \ldots \vee \varphi^{-k+1} \mathfrak{U}\right)
$$




$$
\begin{aligned}
& =\lim _{n \rightarrow \infty} \frac{1}{n}\left[H \left(\mathfrak{U} \vee \varphi^{-1} \mathfrak{U} \vee \ldots \vee \varphi^{-k+1} \mathfrak{U} \vee\left(\varphi^{k}\right)^{-1}\left(\mathfrak{U} \vee \varphi^{-1} \mathfrak{U} \vee \ldots \vee \varphi^{-k+1} \mathfrak{U}\right) \vee \ldots\right.\right. \\
& \left.\left.\ldots \vee\left(\varphi^{k}\right)^{-n+1}\left(\mathfrak{U} \vee \varphi^{-1} \mathfrak{U} \vee \ldots \vee \varphi^{-k+1} \mathfrak{U}\right)\right)\right] \\
& =k \lim _{n \rightarrow \infty} \frac{H\left(\mathfrak{U} \vee \varphi^{-1} \mathfrak{U} \vee \ldots \vee \varphi^{-k+1} \mathfrak{U} \vee \varphi^{-k} \mathfrak{U} \vee \ldots \vee \varphi^{-2 k+1} \mathfrak{U} \vee \ldots \vee \varphi^{-(n-1) k \mathfrak{U} \vee \ldots \vee \varphi^{-n k+1} \mathfrak{U}}\right.}{n k} \\
& =k h(\varphi, \mathfrak{U}) .
\end{aligned}
$$$$
\text { Então } h\left(\varphi^{k}\right) \geq k h(\varphi, \mathfrak{U}) \text { para qualquer cobertura } \mathfrak{U} \text {. }
$$$$
\text { Logo, } h\left(\varphi^{k}\right) \geq k h(\varphi) \text {. }
$$

Por outro lado, temos que

$$
\begin{gathered}
\mathfrak{U} \vee\left(\varphi^{k}\right)^{-1} \mathfrak{U} \vee \ldots \vee\left(\varphi^{k}\right)^{-n+1} \mathfrak{U} \prec \mathfrak{U} \vee \varphi^{-1} \mathfrak{U} \vee \ldots \vee \varphi^{-n k+1} \mathfrak{U} . \text { Logo, } \\
h(\varphi, \mathfrak{U})=\lim _{n \rightarrow \infty} \frac{H\left(\mathfrak{U} \vee \varphi^{-1} \mathfrak{U} \vee \ldots \vee \varphi^{-n k+1} \mathfrak{U}\right)}{n k} \geq \lim _{n \rightarrow \infty} \frac{H\left(\mathfrak{U} \vee\left(\varphi^{k}\right)^{-1} \mathfrak{U} \vee \ldots \vee\left(\varphi^{k}\right)^{-n+1} \mathfrak{U}\right)}{n k} \\
=\frac{1}{k} \lim _{n \rightarrow \infty} \frac{H\left(\mathfrak{U} \vee\left(\varphi^{k}\right)^{-1} \mathfrak{U} \vee \ldots \vee\left(\varphi^{k}\right)^{-n+1} \mathfrak{U}\right)}{n}=\frac{1}{k} h\left(\varphi^{k}, \mathfrak{U}\right)
\end{gathered}
$$

E isso implica que $h(\varphi, \mathfrak{U}) \geq \frac{1}{k} h\left(\varphi^{k}, \mathfrak{U}\right)$, para qualquer cobertura aberta $\mathfrak{U}$.

Assim $k h(\varphi) \geq h\left(\varphi^{k}\right)$.

Portanto $h\left(\varphi^{k}\right)=k h(\varphi)$. 
Corolário 2.2.1. Se $\varphi$ é um homeomorfismo, então $h\left(\varphi^{k}\right)=|k| h(\varphi)$; para qualquer inteiro $k$.

Demonstração: Se $k \geq 0$ segue diretamente do Teorema 2.2.2.

Se $k<0$ temos que $-k>0$, assim pelo Teorema 2.2 .2 temos que $h\left(\varphi^{k}\right)=$ $k h(\varphi)$, mas $-k=|k|$ já que $k<0$, logo $h\left(\varphi^{k}\right)=|k| h(\varphi), \forall k$ inteiro.

Teorema 2.2.3. Sejam $X$ e $Y$ dois espaços topológicos compactos e $\varphi_{1}: X \rightarrow X$ $e \varphi_{2}: Y \rightarrow Y$ aplicações contínuas. Então

$$
h\left(\varphi_{1} \times \varphi_{2}\right)=h\left(\varphi_{1}\right)+h\left(\varphi_{2}\right)
$$

onde $\left(\varphi_{1} \times \varphi_{2}\right): X \times Y \rightarrow X \times Y$ definida por $\left(\varphi_{1} \times \varphi_{2}\right)(x, y)=\left(\varphi_{1}(x), \varphi_{2}(y)\right)$.

Demonstração: Coberturas abertas de $X \times Y$ da forma $\mathfrak{U} \times \mathfrak{B} \equiv\{A \times B$ tal que $A \in \mathfrak{U}, B \in \mathfrak{B}\}$ têm a propriedade que $N_{X \times Y}(\mathfrak{U} \times \mathfrak{B})=N_{X}(\mathfrak{l}) \cdot N_{Y}(\mathfrak{B})$ e $(\mathfrak{U} \times \mathfrak{B}) \vee\left(\mathfrak{U}^{\prime} \times \mathfrak{B}^{\prime}\right)=\left(\mathfrak{U} \vee \mathfrak{U}^{\prime}\right) \times\left(\mathfrak{B} \vee \mathfrak{B}^{\prime}\right)$, onde $\mathfrak{U}, \mathfrak{U}^{\prime}$ são coberturas de $X$ e $\mathfrak{B}, \mathfrak{B}^{\prime}$ são coberturas de $Y$.

De fato:

Provemos inicialmente que $N_{X \times Y}(\mathfrak{U} \times \mathfrak{B})=N_{X}(\mathfrak{l}) \cdot N_{Y}(\mathfrak{B})$.

Escolhamos subcobertura de $\mathfrak{U}$ minimal para $X$, ou seja, ela tem $N_{X}(\mathfrak{U})$ elementos, que denotaremos por $A_{1}, \ldots, A_{N_{X}(\mathfrak{l l})}$. Escolhamos também subcobertura de $\mathfrak{B}$ minimal para $Y$, que denotaremos por $B_{1}, \ldots, B_{N_{X}(\mathfrak{B})}$. Então $\left(A_{i} \times B_{j}\right)_{(i, j)}$ é cobertura de $X \times Y$, possui $N_{X}(\mathfrak{U}) \cdot N_{Y}(\mathfrak{B})$ elementos é uma subcobertura de $\mathfrak{U} \times \mathfrak{B}$. Portanto temos que $N_{X \times Y}(\mathfrak{U} \times \mathfrak{B}) \leq N_{X}(\mathfrak{U}) \cdot N_{Y}(\mathfrak{B})$.

Verifiquemos que nenhum elemento pode ser eliminado:

Considere o elemento $A_{\mathfrak{i}_{0}} \times B_{\mathfrak{i}_{0}}$ dessa subcobertura de $\mathfrak{U} \times \mathfrak{B}$, temos que: $\exists x_{i_{0}} \in A_{i_{0}}$ e $x_{i_{0}} \notin A_{i}, i \neq i_{0}$ (senão $A_{i}$ não seria minimal) $\exists y_{j_{0}} \in B_{j_{0}}$ e $y_{j_{0}} \notin B_{j}, j \neq j_{0}$ (senão $B_{j}$ não seria minimal). 
Portanto, o elemento $\left(x_{i_{0}}, y_{j_{0}}\right) \in A_{i_{0}} \times B_{j_{0}}$ não pertence a nenhum outro $A_{i} \times B_{j}$, isto é, nenhum elemento dessa subcobertura de $\mathfrak{U} \times \mathfrak{B}$ pode ser eliminado. Portanto $N_{X \times Y}(\mathfrak{U} \times \mathfrak{B})=N_{X}(\mathfrak{U}) . N_{Y}(\mathfrak{B})$.

Provemos agora que $(\mathfrak{U} \times \mathfrak{B}) \vee\left(\mathfrak{U}^{\prime} \times \mathfrak{B}^{\prime}\right)=\left(\mathfrak{U} \vee \mathfrak{U}^{\prime}\right) \times\left(\mathfrak{B} \vee \mathfrak{B}^{\prime}\right)$

De fato:

Qualquer membro de $(\mathfrak{U} \times \mathfrak{B}) \vee\left(\mathfrak{U}^{\prime} \times \mathfrak{B}^{\prime}\right)$ é da forma $(A \times B) \cap\left(A^{\prime} \times B^{\prime}\right)$, onde $(A \times B) \in \mathfrak{U} \times \mathfrak{B}$ e $\left(A^{\prime} \times B^{\prime}\right) \in \mathfrak{U}^{\prime} \times \mathfrak{B}^{\prime}$, porém $(A \times B) \cap\left(A^{\prime} \times B^{\prime}\right)=\left(A \cap A^{\prime}\right) \times$ $\left(B \cap B^{\prime}\right)$, onde $\left(A \cap A^{\prime}\right) \in\left(\mathfrak{U} \vee \mathfrak{U}^{\prime}\right)$ e $\left(B \cap B^{\prime}\right) \in\left(\mathfrak{B} \vee \mathfrak{B}^{\prime}\right) \Rightarrow(\mathfrak{U} \times \mathfrak{B}) \vee\left(\mathfrak{U}^{\prime} \times \mathfrak{B}^{\prime}\right) \subset$ $\left(\mathfrak{U} \vee \mathfrak{U}^{\prime}\right) \times\left(\mathfrak{B} \vee \mathfrak{B}^{\prime}\right)$

Analogamente, qualquer membro de $\left(\mathfrak{U} \vee \mathfrak{U}^{\prime}\right) \times\left(\mathfrak{B} \vee \mathfrak{B}^{\prime}\right)$ é da forma $\left(A \cap A^{\prime}\right) \times$ $\left(B \cap B^{\prime}\right)=(A \times B) \cap\left(A^{\prime} \times B^{\prime}\right)$ e assim temos $\left(\mathfrak{U} \vee \mathfrak{U}^{\prime}\right) \times\left(\mathfrak{B} \vee \mathfrak{B}^{\prime}\right) \subset(\mathfrak{U} \times \mathfrak{B}) \vee\left(\mathfrak{U}^{\prime} \times \mathfrak{B}^{\prime}\right)$

Como $N_{X \times Y}(\mathfrak{U} \times \mathfrak{B})=N_{X}(\mathfrak{U}) \cdot N_{Y}(\mathfrak{B})$, temos que $H_{X \times Y}(\mathfrak{U} \times \mathfrak{B})=H_{X}(\mathfrak{U})+$ $H_{Y}(\mathfrak{B})$.

Consequentemente,

$$
h\left(\varphi_{1} \times \varphi_{2}, \mathfrak{U} \times \mathfrak{B}\right)=h\left(\varphi_{1}, \mathfrak{U}\right)+h\left(\varphi_{2}, \mathfrak{B}\right) .
$$

Observe que do lado esquerdo da igualdade temos uma cobertura de uma dada forma que no caso é o produto cartesiano das duas coberturas, e do lado direito da igualdade temos coberturas genéricas. Logo,

$$
h\left(\varphi_{1} \times \varphi_{2}\right) \geq h\left(\varphi_{1}\right)+h\left(\varphi_{2}\right) .
$$

Para estabelecer a desigualdade no outro sentido precisamos apenas mostrar que para uma cobertura arbitrária $\mathfrak{C}$ de $X \times Y$ existe um refinamento da forma $\mathfrak{U} \times \mathfrak{B}$, onde $\mathfrak{U}$ é uma cobertura de $X$ e $\mathfrak{B}$ é uma cobertura de $Y$. Como todo subconjunto aberto de $X \times Y$ é uma união de conjuntos da forma $A \times B, A$ subconjunto aberto de $X, B$ subconjunto aberto de $Y$, que denominaremos para facilidade "retângulos", escolhemos um refinamento de $\mathfrak{C}$ consistindo apenas de 
"retângulos" abertos e desta escolha obtemos uma subcobertura minimal $\mathfrak{C}^{\prime}$, isto é, $\mathfrak{C}^{\prime}=\left\{A_{1}^{\prime} \times B_{1}^{\prime}, \ldots, A_{N\left(\mathbb{C}^{\prime}\right)}^{\prime} \times B_{N\left(\mathbb{C}^{\prime}\right)}^{\prime}\right\}$ e $\mathfrak{C} \prec \mathfrak{C}^{\prime}$.

Seja $A_{x}$ a intersecção de todos conjuntos de $\mathfrak{U}^{\prime}$ que contém o elemento $x \in X$ e $B_{y}$ a interseç̧ão de todos conjuntos de $\mathfrak{B}^{\prime}$ que contém o elemento $y \in Y$.

Estes conjuntos definidos são ainda abertos, e podemos escolher um número finito de pontos $x_{1}, \ldots, x_{m}$ em $X$ e $y_{1}, \ldots, y_{n}$ em $Y$ tal que

$$
\mathfrak{U}=\left\{A_{x_{1}}, \ldots, A_{x_{m}}\right\} \text { e } \mathfrak{B}=\left\{B_{y_{1}}, \ldots, B_{y_{m}}\right\}
$$

são coberturas de $X$ e $Y$ respectivamente.

Considere qualquer conjunto $A_{x_{\mathfrak{i}}} \times B_{y_{j}} \in \mathfrak{U} \times \mathfrak{B}$. Como $\mathfrak{C}^{\prime}$ é uma cobertura de $X \times Y,\left(x_{i}, y_{j}\right) \in A_{k}^{\prime} \times B_{k}^{\prime}$ para algum inteiro $k$ entre 1 e $N\left(\mathfrak{C}^{\prime}\right)$, e portanto, $x_{i} \in A_{k}^{\prime}$ e $y_{j} \in B_{k}^{\prime}$. Segue que $A_{x_{i}} \subset A_{k}^{\prime}$ e $B_{y_{j}} \subset B_{k}^{\prime}$, isto é, $A_{x_{i}} \times B_{y_{j}} \subset A_{k}^{\prime} \times B_{k}^{\prime}$ o que implica que $\mathfrak{C} \prec \mathfrak{C}^{\prime} \prec \mathfrak{U} \times \mathfrak{B}$.

Obtemos, portanto, um refinamento da cobertura genérica $\mathfrak{C}$ por uma cobertura da forma $\mathfrak{U} \times \mathfrak{B}$.

Teorema 2.2.4. Sejam $X_{1}$ e $X_{2}$ dois subconjuntos fechados de $X$ tal que $X=$ $X_{1} \cup X_{2}$ e $\varphi X_{1} \subseteq X_{1}, \varphi X_{2} \subseteq X_{2}$ para $\varphi: X \rightarrow X$ contínua. Então

$$
h(\varphi)=\max \left\{h\left(\varphi_{1}\right), h\left(\varphi_{2}\right)\right\}
$$

onde $\varphi_{1}=\left.\varphi\right|_{X_{1}}$ e $\varphi_{2}=\left.\varphi\right|_{X_{2}}$

Demonstraçāo: Seja $i=1$ ou 2. Para qualquer cobertura aberta $\mathfrak{U}$ de $X$ a família $(\mathfrak{U})_{i}=\left\{A \cap X_{i} \mid A \in \mathfrak{U}\right\}$ define uma cobertura aberta de $X_{i}$, aberta no subespaço topológico $X_{i}$. Empregando índices à $N$ para indicar o espaço cuja cobertura está sendo contada, temos $N_{i}\left((\mathfrak{U})_{i}\right) \leq N(\mathfrak{U})$.

Para coberturas abertas $\mathfrak{U}$ e $\mathfrak{B}$ de $X$ temos também $(\mathfrak{U} \vee \mathfrak{B})_{\mathfrak{i}}=(\mathfrak{U})_{\mathfrak{i}} \vee(\mathfrak{B})_{i}$, pois, para $C \in(\mathfrak{U} \vee \mathfrak{B})_{i}$, temos que $C=(A \cap B) \cap X_{\mathfrak{i}}$, onde $(A \cap B) \in \mathfrak{U} \vee \mathfrak{B}$. Mas $C=(A \cap B) \cap X_{i}=\left(A \cap X_{i}\right) \cap\left(B \cap X_{i}\right) \in(\mathfrak{U})_{i} \vee(\mathfrak{B})_{i}$. Além disso, $\varphi_{i}^{-1}(\mathfrak{U})_{i}=\left(\varphi^{-1} \mathfrak{U}\right)_{i}$. Seja $\mathfrak{U}_{i}$ uma cobertura aberta arbitrária de $X_{i}$, aberta no subespaço topológico $X_{i}$ (cada elemento $B$ desta cobertura é da forma $A \cap X_{i}$, 
onde $A$ é aberto de $X$ ). Considerando estes $A$ 's e mais o aberto $X-A_{i}$ obtemos uma cobertura aberta $\mathfrak{U}$ de $X$ tal que $(\mathfrak{U})_{i}=\mathfrak{U}_{i}$, isto é, $\mathfrak{U}=\mathfrak{U}_{i}^{*} \cup\left(X-X_{i}\right)$, onde os elementos de $\mathfrak{U}_{i}^{*}$ são abertos de $X$ que interceptados com $X_{i}$ nos definem os abertos de $\mathfrak{U}_{i}$.

$$
N_{i}\left(\bigvee_{k=0}^{n-1} \varphi_{i}^{-k} \mathfrak{U}_{i}\right)=N_{i}\left(\bigvee_{k=0}^{n-1}\left(\varphi^{-k} \mathfrak{U}\right)_{i}\right)=N_{i}\left(\left(\bigvee_{k=0}^{n-1} \varphi^{-k} \mathfrak{U}\right)_{i}\right) \leq N\left(\bigvee_{k=0}^{n-1} \varphi^{-k} \mathfrak{U}\right)
$$

Portanto, $N_{i}\left(\bigvee_{k=0}^{n-1} \varphi_{i}^{-k} \mathfrak{U}_{i}\right) \leq N\left(\bigvee_{k=0}^{n-1} \varphi^{-k} \mathfrak{U}\right)$

$\operatorname{Assim} h\left(\varphi_{i}, \mathfrak{U l}_{i}\right) \leq h(\varphi, \mathfrak{U})$.

Logo $h\left(\varphi_{i}\right) \leq h(\varphi)$.

Por outro lado, para qualquer cobertura aberta $\mathfrak{U}$ de $X$ temos

$$
N\left(\bigvee_{k=0}^{n-1} \varphi^{-k} \mathfrak{U}\right) \leq N_{1}\left(\bigvee_{k=0}^{n-1}\left(\varphi^{-k} \mathfrak{U}\right)_{1}\right)+N_{2}\left(\bigvee_{k=0}^{n-1}\left(\varphi^{-k} \mathfrak{U}\right)_{2}\right)
$$

e como anteriormente

$$
\left.N_{i}\left(\left(\bigvee_{k=0}^{n-1} \varphi^{-k} \mathfrak{U}\right)\right)_{i}\right) \doteq N_{i}\left(\bigvee_{k=0}^{n-1} \varphi_{i}^{-k}(\mathfrak{U})_{i}\right), i=1,2
$$

assim,

$$
N\left(\bigvee_{k=0}^{n-1} \varphi^{-k} \mathfrak{U}\right) \leq N_{1}\left(\bigvee_{k=0}^{n-1} \varphi_{1}^{-k}(\mathfrak{U})_{1}\right)+N_{2}\left(\bigvee_{k=0}^{n-1} \varphi_{2}^{-k}(\mathfrak{U})_{2}\right)
$$

Consequentemente,

$$
\log N\left(\bigvee_{k=0}^{n-1} \varphi^{-k} \mathfrak{U}\right) \leq \log \left[N _ { 1 } \left(\bigvee_{k=0}^{n-1}\left(\varphi_{1}^{-k}(\mathfrak{U})_{1}\right)+N_{2}\left(\bigvee_{k=0}^{n-1}\left(\varphi_{2}^{-k}(\mathfrak{U})_{2}\right)\right]\right.\right.
$$

Agora passando o limite e aplicando o Lema 1.0.7, temos 


$$
h(\varphi, \mathfrak{U}) \leq \max \left\{h\left(\varphi_{1},(\mathfrak{U})_{1}\right), h\left(\varphi_{2},(\mathfrak{U})_{2}\right)\right\} .
$$

Tomando o supremo, temos

$$
h(\varphi)=\max \left\{h\left(\varphi_{1}\right), h\left(\varphi_{2}\right)\right\}
$$

Teorema 2.2.5. Seja $\sim$ uma relação de equivalência sobre um conjunto compacto $X$. Seja $\varphi: X \rightarrow X$ uma aplicação contínua, tal que se $x \sim y \Rightarrow \varphi(x) \sim$ $\varphi(y)$. Se $\bar{\varphi}: X / \sim \rightarrow X / \sim$ definida por $\tilde{\varphi} \pi=\pi \varphi$ onde $\pi: X \rightarrow X / \sim$ é a projeção, então

$$
h(\tilde{\varphi}) \leq h(\varphi)
$$

Demonstração: Seja $\tilde{\mathfrak{U}}$ uma cobertura aberta de $X / \sim$. Então $\pi^{-1} \tilde{\mathfrak{U}}$ é uma cobertura aberta de $X$, já que $\pi$ é contínua e sobrejetora. Assim

$$
N_{X}\left(\pi^{-1} \tilde{\mathfrak{U}}\right)=N_{X / \sim}(\tilde{\mathfrak{U}})
$$

Portanto,

$$
h\left(\varphi, \pi^{-1} \tilde{\mathfrak{U}}\right)=h(\tilde{\varphi}, \tilde{\mathfrak{U}})
$$

Logo,

$$
h(\varphi)=\sup _{\mathfrak{U}} h(\dot{\varphi}, \mathfrak{U}) \geq \sup _{\tilde{\mathfrak{U}}} h\left(\varphi, \pi^{-1} \tilde{\mathfrak{U}}\right)=\sup _{\tilde{\mathfrak{U}}} h(\tilde{\varphi}, \tilde{\mathfrak{U}})=h(\tilde{\varphi}) .
$$

Portanto $h(\varphi) \geq h(\tilde{\varphi})$. 


\subsection{Cálculo de entropia em espaços métricos}

Nesta seção $X$ é um espaço métrico compacto com a métrica $d$.

Definição 2.3.1. $O$ diâmetro $d(\mathfrak{U})$ de uma cobertura $\mathfrak{U}$ de $X$ é definido por

$$
d(\mathfrak{I})=\sup _{A \in \mathfrak{L}} d(A)
$$

onde $d(A)$ é o diâmetro do conjunto $A$.

Proposição 2.3.1. (Lema da Cobertura de Lebesgue) Para toda cobertura aberta $\mathfrak{U}$ de um espaço métrico compacto $X$ existe $\varepsilon>0$ tal que se $A$ é um conjunto com $d(A)<\varepsilon$, entāo $A$ está contida em um dos membros de $\mathfrak{U}$. $O$ supremo de todos tais números $\varepsilon$ é chamado o número de Lebesgue de $\mathfrak{U}$.

Corolário 2.3.1. Se $\mathfrak{U}$ e $\mathfrak{B}$ sāo coberturas abertas de $X$ e se $d(\mathfrak{B})$ é menor que o número de Lebesgue de $\mathfrak{U}$, então $\mathfrak{U} \prec \mathfrak{B}$.

Corolário 2.3.2. Se $\mathfrak{S}_{n}$ é uma sequência de coberturas abertas tal que

(1) $\mathfrak{U}_{n} \prec \mathfrak{U}_{n+1}$,

(2) $d\left(\mathfrak{L}_{n}\right) \rightarrow 0$, quando $n \rightarrow \infty$,

então $\mathfrak{L}_{n}$ é uma sequência de coberturas refinada.

Demonstração: Por definição, uma sequência $\left\{\mathfrak{U}_{n} \mid n=1,2, \ldots\right\}$ de coberturas abertas é refinada se

(1) $\mathfrak{U}_{n} \prec \mathfrak{U}_{n+1}$,

(2) Para toda cobertura aberta $\mathfrak{B}$ existe $\mathfrak{U}_{n}$ tal que $\mathfrak{B} \prec \mathfrak{U}_{n}$.

A primeira condição do Corolário segue da definição. 
Sejam $\mathfrak{B}$ uma cobertura aberta e $\delta$ o seu número de Lebesgue. Como $d\left(\mathfrak{U}_{n}\right) \rightarrow 0, \exists n_{0} \mid d\left(\mathfrak{U}_{n_{0}}\right) \leq \frac{\delta}{2}$, denotamos esta cobertura $\left\{\mathfrak{U}_{n_{0}}^{\delta}\right\}$ e temos $\mathfrak{B} \prec \mathfrak{U}_{n_{0}}^{\delta}$.

Observação 2.3.1. O corolário 2.3.2 assegura a existência de sequências refinadas em espaços métricos. Por exemplo, a sequência $\left\{\mathfrak{U}_{n}\right\}$, onde $\mathfrak{U}_{n}$ é o conjunto de todas as bolas de diâmetro menor que $\frac{1}{n}$, é refinada. Além disso, dada qualquer sequência $\left\{\mathfrak{B}_{n}\right\}$ de coberturas satisfazendo a condição (2) do corolário 2.3.2, podemos construir $\left\{\mathfrak{U}_{n}\right\}$ tal que $\mathfrak{U}_{n}=\bigvee_{k=0}^{n} \mathfrak{B}_{k}$ que satisfaz (1) e (2) e assim é refinada.

Vamos agora dar alguns exemplos do cálculo de entropia de funções em espaços métricos.

Exemplo 2.3.1. Se $\varphi: X \rightarrow X$ é uma isometria (sobrejetora), então $h(\varphi)=0$.

Demonstração: Seja $\mathfrak{U}_{p}$ a família de todos os conjuntos abertos de diâmetro menor que $\frac{1}{p}$. Uma tal família possui a propriedade que $\mathfrak{U}_{p} \vee \mathfrak{U}_{p}=\mathfrak{U}_{p}$, pois qualquer membro de $\mathfrak{U}_{p} \vee \mathfrak{U}_{p}$ é da forma $U \cap U^{\prime}$, onde $U, U^{\prime} \in \mathfrak{U}_{p}$, ou seja, $U \cap U^{\prime}$ tem diâmetro menor que $\frac{1}{p}$, $\log U \cap U^{\prime} \in \mathfrak{U}_{p}$, portanto $\mathfrak{U}_{p} \vee \mathfrak{U}_{p} \subset \mathfrak{U}_{p}$. Analogamente, $\mathfrak{U}_{p} \vee \mathfrak{U}_{p} \supset \mathfrak{U}_{p}$. Como $\varphi$ é uma isometria, $\varphi^{-1} \mathfrak{U}_{p}=\mathfrak{U}_{p}$. Isto implica que $\mathfrak{U}_{p}=\mathfrak{U}_{p} \vee \varphi^{-1} \mathfrak{U}_{p} \vee \ldots \vee \varphi^{-n+1} \mathfrak{U}_{p}$, pois $\varphi^{-1} \mathfrak{U}_{p}=\mathfrak{U}_{p}$ e $\mathfrak{U}_{p} \vee \mathfrak{U}_{p}=\mathfrak{U}_{p}$.

Entāo

$$
\begin{gathered}
h\left(\varphi, \mathfrak{U}_{p}\right)=\lim _{n \rightarrow \infty} \frac{H\left(\mathfrak{U}_{p} \vee \varphi^{-1} \mathfrak{U}_{p} \vee \ldots \vee \varphi^{-n+1} \mathfrak{U}_{p}\right)}{n}=\lim _{n \rightarrow \infty} \frac{H\left(\mathfrak{U}_{p}\right)}{n} \\
\quad=\lim _{n \rightarrow \infty} \frac{\log N\left(\mathfrak{U}_{p}\right)}{n}=0 .
\end{gathered}
$$

Portanto, $h\left(\varphi, \mathfrak{U}_{p}\right)=0$. 
Como $\left\{\bigcup_{p}\right\}$ é uma sequência de coberturas abertas tal que

(1) $\mathfrak{U}_{p} \prec \mathfrak{U}_{p+1}$,

(2) $d\left(\mathfrak{U}_{p}\right) \rightarrow 0$ quando $p \rightarrow \infty$,

temos pelo Corolário 2.3.2, que $\mathfrak{U}_{p}$ é uma sequência refinada.

Assim pela Propriedade 2.1.14, temos que $h(\varphi)=\lim _{p \rightarrow \infty} h\left(\varphi, \mathfrak{U}_{p}\right)=\lim _{p \rightarrow \infty} 0=0$. Logo $h(\varphi)=0$.

Exemplo 2.3.2. Seja $(X, \varphi)$ um sistema dinâmico compacto equicontínuo, então $h(\varphi)=0$.

Demonstração: A métrica $d^{\prime}$ definida por $d^{\prime}(x, y)=\sup _{n \in \mathbf{Z}} d\left(\varphi^{n} x, \varphi^{n} y\right)$ é equivalente a $d$.

a) Provemos que dado $B_{d}\left(x_{0}, r\right)$ existe $B_{d^{\prime}}\left(x_{0}, r\right) \subset B_{d}\left(x_{0}, r\right)$. (Observe que $\left.d^{\prime}(x, y) \geq d(x, y)\right)$.

Dado $x \in B_{d^{\prime}}\left(x_{0}, r\right)=\left\{x \in X \mid d^{\prime}\left(x_{0}, x\right)<r\right\}$ temos que $d^{\prime}\left(x_{0}, x\right)<r \Rightarrow$ $d\left(x_{0}, x\right)<r \Rightarrow x \in B_{d}\left(x_{0}, r\right)$.

b) Verifiquemos agora que $B_{d}\left(x_{0}, r\right) \subset B_{d^{\prime}}\left(x_{0}, \delta\right)$.

Observe que fixado $x_{0}$ podemos construir a função $\xi:(X, d) \rightarrow \mathbb{R}$ dada por $\xi(x)=\left[d^{\prime}\left(x_{0}, x\right)-d\left(x_{0}, x\right)\right]$. Por equicontinuidade $\xi$ é contínua. De fato: Já sabemos que $d\left(x_{0}, x\right)$ é contínua, falta verificar que $d^{\prime}\left(x_{0}, x\right)$ é contínua. Para isso podemos definir a sequência $d^{n}\left(x_{0}, x\right)=\sup _{-n \leq i \leq n} d\left(\varphi^{i}\left(x_{0}\right), \varphi^{i}(x)\right)$ entāo

$$
d^{\prime}\left(x_{0}, x\right)=\lim _{n \rightarrow \infty} d^{n}\left(x_{0}, x\right)=\sup _{i \in \mathbf{Z}} d\left(\varphi^{i}\left(x_{0}\right), \varphi^{i}(x)\right) .
$$

Portanto, $\xi=\lim _{n \rightarrow \infty} \xi^{n}$, onde $\xi^{n}(x)=\left[d^{n}\left(x_{0}, x\right)-d\left(x_{0}, x\right)\right]$.

Observe que $\left.\xi\right|_{D_{d}\left(x_{0}, r\right)}$ tem máximo igual a $t$. Seja $x \in B_{d}\left(x_{0}, r\right) \Rightarrow x \in$ $D_{d}\left(x_{0}, r\right) \Rightarrow d^{\prime}\left(x_{0}, x\right)-d\left(x_{0}, x\right) \leq t \Rightarrow d^{\prime}\left(x_{0}, x\right) \leq d\left(x_{0}, x\right)+t \Rightarrow x \in B_{d^{\prime}}\left(x_{0}, \delta\right)$, onde $\delta=d\left(x_{0}, x\right)+t$.

Assim temos que $d^{\prime}$ é equivalente a $d$. 
Com respeito a essa nova métrica, $\varphi$ é uma isometria:

$$
\begin{gathered}
d^{\prime}(\varphi(x), \varphi(y))=\sup _{-\infty<n<+\infty} d\left(\varphi^{n+1}(x), \varphi^{n+1}(y)\right) \\
d^{\prime}(x, y)=\sup _{-\infty<n<+\infty} d\left(\varphi^{n}(x), \varphi^{n}(y)\right)
\end{gathered}
$$

Sendo $\varphi$ uma isometria temos, pelo Exemplo 2.3.1, que $h(\varphi)=0$.

Exemplo 2.3.3. Seja $X$ um grupo topológico compacto separável e $\varphi: X \rightarrow X$ definida por $\varphi(x)=a x b$, onde $a, b \in X$. Então $h(\varphi)=0$.

Demonstração: $X$ é metrizável, digamos com a métrica $d$. A "rotação" $\varphi$ é uma isometria com respeito a métrica $d^{\prime}$ definida por $d^{\prime}(x, y)=\sup _{u, v \in X} d(u x v, u y v)$ que é equivalente a $d$.

Vejamos que $\varphi$ é isometria:

$$
\begin{aligned}
d^{\prime}(\varphi(x), \varphi(y)) & =\sup _{u, v \in X} d(u \varphi(x) v, u \varphi(y) v)=\sup _{u, v \in X} d(\bar{u} x \bar{v}, \bar{u} y \bar{v}) \\
& =\sup _{\bar{u}, \bar{v} \in X} d(\bar{u} x \bar{v}, \bar{u} y \bar{v})=d^{\prime}(x, y), \text { onde } \bar{u}=\dot{u} a \mathrm{e} \bar{v}=b v .
\end{aligned}
$$

Portanto,

$$
d^{\prime}(\varphi(x), \varphi(y))=d^{\prime}(x, y)
$$

Logo, $d^{\prime}$ é isometria.

Assim, pelo Exemplo 2.3.1, temos que $h(\varphi)=0$.

Exemplo 2.3.4. Seja $X$ o círculo unitário. Se $\varphi: X \rightarrow X$ é um homeomorfismo, então $h(\varphi)=0$.

Demonstração: Seja $\mathfrak{U}_{p}$ uma cobertura de $X$ por intervalos de comprimento de arco $\frac{1}{p}$. A cobertura $\mathfrak{U}_{p} \vee \varphi^{-1} \mathfrak{U}_{p} \vee \ldots \vee \varphi^{-n+1} \mathfrak{U}_{p}$ é uma cobertura de $X$ por intervalos e 


$$
N\left(\mathfrak{U}_{p} \vee \varphi^{-1} \mathfrak{U}_{p} \vee \ldots \vee \varphi^{-n+1} \mathfrak{U}_{p}\right) \leq n N\left(\mathfrak{U}_{p}\right)
$$

Assim,

$$
\begin{gathered}
h\left(\varphi, \mathfrak{U}_{p}\right)=\lim _{n \rightarrow \infty} \frac{H\left(\mathfrak{U}_{p} \vee \varphi^{-1} \mathfrak{U}_{p} \vee \ldots \vee \varphi^{-n+1} \mathfrak{U}_{p}\right)}{n} \\
=\lim _{n \rightarrow \infty} \frac{\log N\left(\mathfrak{U}_{p} \vee \varphi^{-1} \mathfrak{U}_{p} \vee \ldots \vee \varphi^{-n+1} \mathfrak{U}_{p}\right)}{n} \leq \lim _{n \rightarrow \infty} \frac{\log \left(n N\left(\mathfrak{U}_{p}\right)\right)}{n} \\
=\lim _{n \rightarrow \infty} \frac{\left[\log n+\log N\left(\mathfrak{U}_{p}\right)\right]}{n}=\lim _{n \rightarrow \infty}\left[\frac{\log n}{n}+\frac{\log N\left(\mathfrak{U}_{p}\right)}{n}\right]=0 .
\end{gathered}
$$

Logo, $h\left(\varphi, \mathfrak{U}_{p}\right)=0$.

Como $\left\{\mathfrak{U}_{p} \mid p=1,2, \ldots\right\}$ é refinada, temos pela Propriedade 2.1.14 que

$$
h(\varphi)=\lim _{p \rightarrow \infty} h\left(\varphi, \mathfrak{U}_{p}\right)=\lim _{p \rightarrow \infty} 0=0 .
$$

Portanto, $h(\varphi)=0$.

Exemplo 2.3.5. Expressando o espaço das sequências de 'zeros e uns', indexadas em $\mathbb{Z}$, por

$$
X=\prod_{i=-\infty}^{\infty} X_{i}
$$

onde $X_{i}=\{0,1\}$ e $X_{i}$ com a topologia discreta, o espaço $X$ é compacto na topologia do produto cartesiano pelo Teorema de Tychonoff. (Proposição 1.0.6)

Podemos também expressar $X$ como $X=\ldots \times\{0,1\} \times\{0,1\} \times\{0,1\} \times \ldots$ Assim um elemento de $X$ é da forma $(. . ., 1,0, \underline{1}, 0,0, \ldots)$, onde o traço sob um dos valores determina a posição zero. Uma outra forma também usada é como função quase-nula $\phi: \mathbb{Z} \rightarrow \bigcup_{i=-\infty}^{\infty} X_{i}^{*}$, onde $X_{i}^{*}=i \times X$. 
Seja $\left(x_{i}\right)$ a i-ésima componente da sequência $x \in X$. Então a topologia do produto cartesiano sobre $X$ é a mesma que a determinada pela métrica $d$, onde

$$
d(x, y)=\sum_{-\infty}^{\infty} \frac{\left|(x)_{i}-(y)_{i}\right|}{2^{|i|}}
$$

Veja demonstração na Observação 2.3.4 no final desse Exemplo.

Considere o homeomorfismo $\varphi: X \rightarrow X$ chamado o "shift" (translação) $e$ definida por $(\varphi x)_{i}=(x)_{i+1}$. É fácil ver que $\varphi$ é homeomorfismo.

Seja $\mathfrak{U}=\left\{A_{0}, B_{0}\right\}$, onde $A_{0}=\left\{\left(x_{i}\right) \mid(x)_{0}=0\right\}, B_{0}=\left\{\left(x_{i}\right) \mid(x)_{0}=1\right\} e$ $\mathfrak{U}_{p}=\bigvee_{k=-p}^{p} \varphi^{k} \mathfrak{U}, p=0,1,2, \ldots$.

Veremos que $d\left(\mathfrak{U}_{p}\right) \rightarrow 0$ quando $\dot{p} \rightarrow \infty$, e portanto a sequência $\left\{\mathfrak{U}_{p}\right\}$ é refinada. De fato:

Temos que:

$$
\frac{\left|x_{i}-y_{i}\right|}{2^{|i|}} \leq \frac{1}{2^{|i|}} \Rightarrow \sum \frac{\left|x_{i}-y_{i}\right|}{2^{|i|}} \leq \sum \frac{1}{2^{|i|}}
$$

Temos que $A_{0} \cap B_{0}=\emptyset$ e $A_{0} \cup B_{0}=X$. Além disso,

$$
\begin{gathered}
\sum_{i=-\infty, i \neq 0}^{\infty} \frac{1}{2^{|i|}}=\cdots \frac{1}{4}+\frac{1}{2}+\frac{1}{2}+\frac{1}{4}+\cdots=2 . \\
\text { Comod } d(x, y)=\sum_{-\infty}^{\infty} \frac{\left|(x)_{i}-(y)_{i}\right|}{2^{|i|}} \leq \sum_{-\infty}^{\infty} \frac{1}{2^{|i|}} \text { ed } d\left(A_{0}\right)=\sup \left\{d(x, y), x, y \in A_{0}\right\}
\end{gathered}
$$

temos que $d\left(A_{0}\right)=2$ e da mesma forma $d\left(B_{0}\right)=2$.

Assim $d\left(\mathfrak{U}_{0}\right)=\sup \left\{d\left(A_{0}\right), d\left(B_{0}\right)\right\}=2$.

Agora $\mathfrak{U}_{1}=\bigvee_{k=-1}^{\mathfrak{1}} \varphi^{k} \mathfrak{U}=\varphi^{-1} \mathfrak{U} \vee \mathfrak{U} \vee \varphi \mathfrak{U}$, ou seja, teremos $\varphi^{-1} A_{0} \vee A_{0} \vee \varphi A_{0} e \varphi^{-1} B_{0} \vee B_{0} \vee \varphi B_{0}$ (todas as possíveis intersecções).

Temos que:

$d\left(A_{0} \cap \varphi^{-1}\left(A_{0}\right) \cap \varphi\left(A_{0}\right)\right)=\cdots+\frac{1}{4}+\frac{1}{4}+\cdots=1$ ed $d\left(B_{0} \cap \varphi^{-1}\left(B_{0}\right) \cap \varphi\left(B_{0}\right)\right)=$ 
$\cdots+\frac{1}{4}+\frac{1}{4}+\cdots=1$ e portanto $d\left(\mathfrak{U}_{1}\right)=1$.

Para $\mathfrak{U}_{2}=\stackrel{2}{\bigvee}^{k} \varphi^{k} \mathfrak{U}$ teremos

$\varphi^{-2} A_{0} \vee \varphi^{-1} A_{0} \vee A_{0} \vee \varphi A_{0} \vee \varphi^{2} A_{0} e \varphi^{-2} B_{0} \vee \varphi^{-1} B_{0} \vee B_{0} \vee \varphi B_{0} \vee \varphi^{2} B_{0}$.

$\operatorname{Logod}\left(\mathfrak{U}_{2}\right)=0,5$.

$E$ assim sucessivamente, temos que $d\left(\bigcup_{p}\right) \rightarrow 0$ quando $p \rightarrow \infty$.

Como $\left\{\bigcup_{p}\right\}$ é refinada temos que

$$
\begin{gathered}
h(\varphi, \mathfrak{U}) \leq h\left(\varphi, \mathfrak{U}_{p}\right)=\lim _{n \rightarrow \infty} \frac{H\left(\bigvee_{k=0}^{n-1} \varphi^{-k} \mathfrak{U} \mathfrak{U}_{p}\right)}{n} \\
=\lim _{n \rightarrow \infty} \frac{H\left(\bigvee_{k=-p}^{p} \varphi^{k} \mathfrak{U} \vee \bigvee_{k=-p-1}^{p-1} \varphi^{k} \mathfrak{U} \vee \ldots \vee \bigvee_{k=-p-n+1}^{p-n+1} \varphi^{k} \mathfrak{U}\right)}{n} .
\end{gathered}
$$

Da Propriedade 2.1 .5 e dos cálculos feitos na Observação 2.3.2, após esse Exemplo segue que

$$
\begin{gathered}
h\left(\varphi, \mathfrak{U}_{p}\right)=\lim _{n \rightarrow \infty} \frac{H\left(\bigvee_{k=-p-n+1}^{p} \varphi^{k} \mathfrak{U}\right)}{n}=\lim _{n \rightarrow \infty} \frac{H\left(\bigvee_{k=-p-n+1}^{-p} \varphi^{k} \mathfrak{U}\right)}{n} \\
=\lim _{n \rightarrow \infty} \frac{H\left(\bigcup_{k=-n+1}^{0} \varphi^{k} \mathfrak{U}\right)}{n}=h(\varphi, \mathfrak{U}) \\
\Rightarrow h\left(\varphi, \mathfrak{U}_{p}\right)=h(\varphi, \mathfrak{U}) .
\end{gathered}
$$


Os "cálculos" feitos na Observação 2.3.3 mostram que

$$
N\left(\bigvee_{k=0}^{-n+1} \varphi^{k} \mathfrak{U}\right)=2^{n}
$$

Portanto,

$$
\begin{aligned}
h(\varphi, \mathfrak{U}) & =\lim _{n \rightarrow \infty} \frac{\log N\left(\bigvee_{k=0}^{n+1} \varphi^{-k} \mathfrak{U}\right)}{n}=\lim _{n \rightarrow \infty} \frac{\log 2^{n}}{n}=\lim _{n \rightarrow \infty} \frac{n \log 2}{n}= \\
& =\lim _{n \rightarrow \infty} \log 2=\log 2,
\end{aligned}
$$

isto é,

$$
h(\varphi, \mathfrak{l})=\log 2 .
$$

Segue da Propriedade 2.1.14 e do fato que a sequência $\left\{\mathfrak{U}_{p}\right\}$ é refinada que

$$
h(\varphi)=\log 2
$$

$\operatorname{pois} h(\varphi)=\lim _{p \rightarrow \infty} h\left(\varphi, \mathfrak{L}_{p}\right)$.

Observação 2.3.2. Temos que $\mathfrak{U}_{p}=\varphi^{-p \mathfrak{U} \vee} \varphi^{-p+1} \mathfrak{U} \vee \ldots \vee \mathfrak{U} \vee \varphi \mathfrak{U} \vee \ldots \vee \varphi^{p \mathfrak{U}}$

\section{Agora}

$$
\bigvee_{k=-p}^{p} \varphi^{k} \mathfrak{U} \vee \bigvee_{k=-p-1}^{p-1} \varphi^{k} \mathfrak{U} \vee \ldots \vee \bigvee_{k=-p-n+1}^{p-n+1} \varphi^{k} \mathfrak{U}=
$$

$$
\begin{aligned}
& =\left[\varphi^{-p} \mathfrak{U} \vee \varphi^{-p+1} \mathfrak{U} \vee \ldots \vee \mathfrak{U} \vee \varphi \mathfrak{U} \vee \ldots \vee \varphi^{p-1} \mathfrak{U} \vee \varphi^{p} \mathfrak{U}\right] \vee\left[\varphi^{-p-1} \mathfrak{U} \vee \varphi^{-p} \mathfrak{U} \vee \ldots \vee \mathfrak{U} \vee\right. \\
& \left.\varphi \mathfrak{U} \vee \ldots \vee \varphi^{p-1}\right] \vee \ldots \vee\left[\varphi^{-p-n+1} \mathfrak{U} \vee \varphi^{-p-n+2} \mathfrak{U} \vee \ldots \vee \mathfrak{U} \vee \varphi \mathfrak{U} \vee \ldots \vee \varphi^{p-n+1} \mathfrak{U}\right]=
\end{aligned}
$$$$
=\bigvee_{k=-p-n+1}^{p} \varphi^{k} \mathfrak{U} \mathfrak{l}=\varphi^{-p-n+1} \mathfrak{U} \vee \ldots \vee \varphi^{p} \mathfrak{U}
$$

Além disso, $\bigvee_{k=-p-n+1}^{-p} \varphi^{k} \mathfrak{U} \prec \bigvee_{k=-n+1}^{0} \varphi^{k} \mathfrak{U l}$. 
Observação 2.3.3. Mostremos que $N\left(\bigvee_{k=0}^{-n+1} \varphi^{k} \mathfrak{U}\right)=2^{n}$. Precisamos calcular $N\left(\mathfrak{U} \vee \varphi^{-1} \vee \ldots \vee \varphi^{-n+1} \mathfrak{U}\right)$

Temos:

$$
\begin{array}{lll}
\mathfrak{U}=\left\{A_{0}, B_{0}\right\} & A_{0}=\{* \ldots * \underline{0} * \ldots *\} & B_{0}=\{* \ldots * \underline{1} * \ldots *\} \\
\varphi^{-1} \mathfrak{U}=\left\{A_{1}, B_{1}\right\} & A_{1}=\{* \ldots * 0 \underline{0} * \ldots *\} & B_{1}=\{* \ldots * 1 * \ldots * \ldots *\} \\
\ldots & \\
\varphi^{-n+1} \mathfrak{U}=\left\{A_{-n+1}, B_{-n+1}\right\} & A_{-n+1}=\{* \ldots 0 \ldots * \underline{*} \ldots *\} & B_{-n+1}=\{* \ldots 1 \ldots * \underline{*} \ldots *\} \\
\downarrow & \downarrow \\
& (-n+1) \text {-posição } & (-n+1) \text {-posição }
\end{array}
$$

Logo a intersecção de $n$ elementos genéricos, usando apenas um de cada familia (de dois) acima, terá a forma:

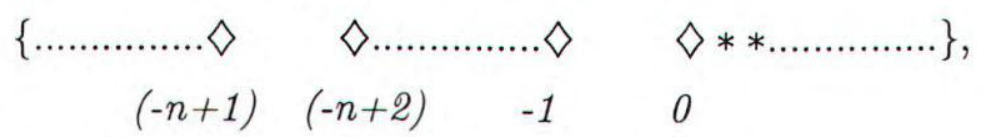

onde nas posições marcadas acima podemos escolher 0 ou 1, conforme a interseç̧ão que escolhermos.

Temos portanto $2^{n}$ escolhas, o que nos dará $N\left(\mathfrak{U} \vee \varphi^{-1} \vee \ldots \vee \varphi^{-n+1} \mathfrak{U}\right)=2^{n}$.

Observação 2.3.4. Provemos que a topologia produto sobre $X$ é a mesma que a determinada pela métrica $d$, onde

$$
d(x, y)=\sum_{-\infty}^{\infty} \frac{\left|(x)_{i}-(y)_{i}\right|}{2^{|i|}}
$$

Demonstração: Seja $\mathfrak{C}_{p}$ a topologia produto em $X$ e seja $\mathfrak{C}_{d}$ a topologia gerada pela métrica $d$.

(i) Mostremos que $\mathfrak{C}_{p} \subset \mathfrak{C}_{d}$.

Dado $\mathfrak{U}$ um aberto básico de $X$ na topologia produto, temos que :

$$
\mathfrak{U}=\prod_{i=-\infty}^{\infty} A_{i}
$$


onde $A_{\alpha_{1}}, \ldots, A_{\alpha_{n}}$ são abertos em $X_{\alpha_{1}}, \ldots, X_{\alpha_{n}}$ respectivamente e $A_{i}=X_{i}$, para $i \neq \alpha_{j}$. Dado um $x \in \mathfrak{U}$, então $(x)_{i} \in A_{i}$ para cada $i$. Seja $\varepsilon<\min \frac{1}{2^{\frac{\alpha}{\alpha_{j}}}, j}, j=1, \ldots, n$.

Afirmamos que $x \in B(x, \varepsilon) \subset \mathfrak{U}$.

De fato, dado $y \in B(x, \varepsilon)$, temos que

$$
d(x, y)<\varepsilon \Rightarrow \sum_{-\infty}^{\infty} \frac{\left|(x)_{i}-(y)_{i}\right|}{2^{|i|}}<\varepsilon \Rightarrow \frac{\left|(x)_{i}-(y)_{i}\right|}{2^{|i|}}<\varepsilon, \forall i .
$$

Em particular,

$$
\frac{\left|(x)_{\alpha_{j}}-(y)_{\alpha_{j}}\right|}{2^{\left|\alpha_{j}\right|}}<\varepsilon
$$

para $j=1, \ldots, n$. Então, como $\left|(x)_{\alpha_{j}}-(y)_{\alpha_{j}}\right|=0$ ou 1 , temos que se $(x)_{\alpha_{j}} \neq(y)_{\alpha_{j}}$ para algum $j=1, \ldots, n$, então $\frac{1}{2^{\left|\alpha_{j}\right|}}=\frac{\left|(x)_{\alpha_{j}}-(y)_{\alpha_{j}}\right|}{2^{\left|\alpha_{j}\right|}}<\varepsilon$, absurdo, pois $\varepsilon<\min \frac{1}{2^{\alpha_{j}} \mid}, j=1, \ldots, n$. Portanto, $(x)_{\alpha_{j}}=(y)_{\alpha_{j}}, \forall j=1, \ldots, n$. Logo, $(y)_{\alpha_{j}} \in A_{\alpha_{j}}, j=1, \ldots, n$ e como $A_{i}=X_{i}$, para $i \neq \alpha_{j}$ temos que $(y)_{i} \in A_{i}, \forall i$.

Portanto, $y \in \mathfrak{U}$ e assim $B(x, \varepsilon) \subset \mathfrak{U}, \log 0 \mathfrak{C}_{p} \subset \mathfrak{C}_{d}$.

(ii) Mostremos agora que $\mathfrak{C}_{d} \subset \mathfrak{C}_{p}$.

Seja um aberto básico $B(x, \varepsilon)$ de $X$ na topologia da métrica.

Como $\sum_{i=0}^{+\infty} \frac{1}{2^{|i|}}$ e $\sum_{i=-\infty}^{0} \frac{1}{2^{|i|}}$ convergem, $\exists N>0$ tal que $\sum_{i=N}^{+\infty} \frac{1}{2^{|i|}}<\frac{\varepsilon}{2}$ e $\sum_{i=-\infty}^{N} \frac{1}{2^{|i|}}<\frac{\varepsilon}{2}$.

Assim

$$
\sum_{|i| \geq N+1} \frac{1}{2^{|i|}}<\varepsilon
$$

Considere

$$
\mathfrak{U}=\prod_{i=-\infty}^{\infty} A_{i}
$$


onde $A_{i}=X_{i}$, para $i \notin\{-N, \ldots, N\}$ e $A_{j}=\left\{(x)_{j}\right\}$ para $j \notin\{-N, \ldots, N\}$.

Então $\mathfrak{U}$ é um aberto na Topologia Produto e $x \in \mathfrak{U}$.

Mostremos que $\mathfrak{U} \subset B(x, \varepsilon)$.

De fato: Dado $y \in U$, temos que $(y)_{j}=(x)_{j}$ para $j \in\{-N, \ldots, N\}$. Assim $\left|(x)_{j}-(y)_{j}\right|=0$ para $j \in\{-N, \ldots, N\}$. Logo,

$$
d(x, y)=\sum_{i=-\infty}^{\infty} \frac{\left|(x)_{i}-(y)_{i}\right|}{2^{|i|}}=\sum_{|i| \geq N+1} \frac{\left|(x)_{i}-(y)_{i}\right|}{2^{|i|}} \leq \sum_{|i| \geq N+1} \frac{1}{2^{|i|}}<\varepsilon,
$$

por $\left(^{*}\right)$.

Portanto $y \in B(x, \varepsilon) \Rightarrow \mathfrak{U} \subset B(x, \varepsilon)$.

Logo, $\mathfrak{C}_{d} \subset \mathfrak{C}_{p}$.

Por (i) e (ii) temos $\mathfrak{C}_{p}=\mathfrak{C}_{d}$.

Observação 2.3.5. Se $X_{i}=\{0,1, \ldots, N-1\}$ no exemplo anterior, então $h(\varphi)=$ $\log N$. Além disso, se $X_{\boldsymbol{i}}$ é algum espaço compacto Hausdorff contendo um número infinito de pontos, então $h(\varphi)=\infty$.

Exemplo 2.3.6. Seja $X$ um toro de dimensão 2, isto é, $X=E^{2} / \sim$, onde $E^{2}$ é o plano euclidiano $e \sim$ é a relação de equivalência que identifica dois pontos no plano se suas coordenadas correspondentes diferem por inteiros. A métrica sobre $X$ pode ser definida em termos da métrica sobre $E^{2}$ tomando a distância entre dois pontos de $X$ como a menor distância entre quaisquer representativos destes pontos em $E^{2}$. Um automorfismo contínuo de grupos $\varphi$ de $X$ tem uma representação $\varphi:(x, y) \rightarrow(a x+b y, c x+d y)\left(\operatorname{adiçãa~mod~1)~onde~}\left(\begin{array}{ll}a & b \\ c & d\end{array}\right)\right.$ é uma matriz unimodular $A$, isto é, uma matriz de inteiros com determinante \pm 1 . Suponha que $A$ tenha dois vetores característicos linearmente independentes $\alpha, \beta$, associados com valores característicos $\lambda$ e $\mu$ onde $|\lambda| \geq 1$. Então $h(\varphi)=\log |\lambda|$. 
Demonstraçāo: Considere uma cobertura de $E^{2}$ por todos os paralelogramos abertos com lados paralelos a $\alpha$ e $\beta$ e tendo comprimento $\frac{1}{p}$. Cada conjunto é um representativo de uma classe de equivalência de conjuntos em relação a $\sim$. Seja $\mathfrak{U}_{p}$ uma cobertura aberta de $X$ por estas classes de equivalência. Se $A$ é um dos paralelogramos acima, então $\varphi^{-n} A$ é equivalente a um paralelogramo tendo lados de comprimento $\frac{|\lambda|^{n}}{p}$ e $\frac{|\lambda|^{-n}}{p}$ que são novamente paralelos aos vetores característicos. Considerando um paralelogramo equivalente a um dos conjuntos de $\mathfrak{U}_{p}, p>1$, podemos ver que "precisamos $|\lambda|^{n-1}$ " paralelogramos para cobri-lo, que são equivalentes aos conjuntos em $\varphi^{-n+\mathfrak{L}_{p}}$. Assim

$$
\begin{gathered}
p^{2}|\lambda|^{n-1} \leq N\left(\mathfrak{U}_{p} \vee \varphi^{-1} \mathfrak{U}_{p} \vee \ldots \vee \varphi^{-n+1} \mathfrak{U}_{p}\right) \leq|\lambda|^{n-1} N\left(\mathfrak{U}_{p}\right) \\
\Rightarrow \log p^{2}|\lambda|^{n-1} \leq \log N\left(\mathfrak{U}_{p} \vee \varphi^{-1} \mathfrak{U}_{p} \vee \ldots \vee \varphi^{-n+1} \mathfrak{U}_{p}\right) \leq \log |\lambda|^{n-1} N\left(\mathfrak{U}_{p}\right) \\
\Rightarrow 2 \log p+(n-1) \log |\lambda| \leq \log N\left(\mathfrak{U}_{p} \vee \varphi^{-1} \mathfrak{U}_{p} \vee \ldots \vee \varphi^{-n+1} \mathfrak{U}_{p}\right) \leq(n-1) \log |\lambda|+\log N\left(\mathfrak{U}_{p}\right) \\
\Rightarrow \lim _{n \rightarrow \infty}\left(\frac{2 \log p}{n}+\frac{(n-1) \log |\lambda|}{n}\right) \leq \lim _{n \rightarrow \infty} \frac{\log N\left(\mathfrak{U}_{p} \vee \varphi^{-1} \mathfrak{U}_{p} \vee \ldots \vee \varphi^{-n+1} \mathfrak{U}_{p}\right)}{n} \\
\leq \lim _{n \rightarrow \infty}\left(\frac{(n-1) \log |\lambda|}{n}+\frac{\log N\left(\mathfrak{U}_{p}\right)}{n}\right) \\
\Rightarrow \log |\lambda| \leq h\left(\varphi, \mathfrak{U}_{p}\right) \leq \log |\lambda| \\
\Rightarrow h\left(\varphi, \mathfrak{U}_{p}\right)=\log |\lambda| \operatorname{para} p>1 .
\end{gathered}
$$

Como $\left\{\mathfrak{U}_{p} \mid p=1,2, \ldots\right\}$ é uma sequência refinada temos

$$
\begin{gathered}
h(\varphi)=\lim _{p \rightarrow \infty} h\left(\varphi, \mathfrak{U}_{p}\right)=\lim _{p \rightarrow \infty} \log |\lambda|=\log |\lambda| \\
\Rightarrow h(\varphi)=\log |\lambda| .
\end{gathered}
$$


Observação 2.3.6. Se $X$ é um toro $n$-dimensional e $\phi$ um automorfismo de $X$ determinado por uma matriz $n \times n$ unimodular tendo valores característicos reais $\lambda_{1}, \ldots, \lambda_{n}$ e $n$ vetores característicos linearmente independentes, então com um argumento similar temos

$$
h(\varphi)=\sum_{|\lambda| \geq 1} \log |\lambda| .
$$

Uma curiosidade baseada nas técnicas deste trabalho é a seguinte:

Proposiçāo 2.3.2. Seja $X$ um espaço métrico compacto com um número infinito de pontos. Seja $\varphi: X \rightarrow X$ uma aplicação contínua. Para qualquer cobertura aberta $\mathfrak{U}$ existe um número $\delta>0$ (dependendo de $\mathfrak{U}$ e $\varphi$ ) tal que

$$
d\left(\varphi^{-1} \mathfrak{U} \vee \varphi^{-2} \mathfrak{U} \vee \ldots \vee \varphi^{-n} \mathfrak{U}\right) \geq \delta>0, \text { para todo } n .
$$

Demonstração: Suponhamos $d\left(\varphi^{-1} \mathfrak{U} \vee \varphi^{-2} \mathfrak{U} \vee \ldots \vee \varphi^{-n} \mathfrak{U}\right) \rightarrow 0$ quando $n \rightarrow \infty$. Existe um inteiro $N$ tal que se $n \geq N$ então $d\left(\varphi^{-1} \mathfrak{U} \vee \varphi^{-2} \mathfrak{U} \vee \ldots \vee \varphi^{-n} \mathfrak{U}\right)<$ número de Lebesgue de $\mathfrak{U}$. Portanto $\mathfrak{U} \prec \varphi^{-1} \mathfrak{U} \vee \varphi^{-2} \mathfrak{U} \vee \ldots \vee \varphi^{-n} \mathfrak{U}, n \geq N$ (Corolário do Lema Cob. de Lebesgue 2.3.1).

Assim $N\left(\mathfrak{U} \vee \varphi^{-1} \mathfrak{U} \vee \varphi^{-2} \mathfrak{U} \vee \ldots \vee \varphi^{-n} \mathfrak{U}\right)=N\left(\varphi^{-1} \mathfrak{U} \vee \varphi^{-2} \mathfrak{U} \vee \ldots \vee \varphi^{-n} \mathfrak{U}\right)$ (pela Propriedade 2.1.5). Mas pela Propriedade 2.1.9, $N\left(\varphi^{-1} \mathfrak{U} \vee \varphi^{-2} \mathfrak{U} \vee \ldots \vee \varphi^{-n} \mathfrak{U}\right)=$ $N\left(\mathfrak{U} \vee \varphi^{-1} \mathfrak{U} \vee \varphi^{-2} \mathfrak{U} \vee \ldots \vee \varphi^{-n+1} \mathfrak{U}\right)$

Por indução, $N\left(\mathfrak{U} \vee \varphi^{-1} \mathfrak{U} \vee \varphi^{-2} \mathfrak{U} \vee \ldots \vee \varphi^{-n} \mathfrak{U}\right)=N\left(\mathfrak{U} \vee \varphi^{-1} \mathfrak{U} \vee \varphi^{-2} \mathfrak{U} \vee \ldots \vee \varphi^{-N} \mathfrak{U}\right)$, para $n \geq N$; isto é, $N\left(\mathfrak{U} \vee \varphi^{-1} \mathfrak{U} \vee \varphi^{-2} \mathfrak{U} \vee \ldots \vee \varphi^{-n} \mathfrak{U}\right)$ é limitado, digamos pelo número $M$. Escolha $M+1$ pontos distintos $x_{1}, \ldots, x_{M+1}$ e seja $n$ tão grande que $d\left(\mathfrak{U} \vee \varphi^{-1} \mathfrak{U} \vee \varphi^{-2} \mathfrak{U} \vee \ldots \vee \varphi^{-n} \mathfrak{U}\right)<\min _{1 \leq i<j \leq M+1} d\left(x_{i}, x_{j}\right)$. Isto é uma contradição, pois para cobrir $x_{1}, \ldots, x_{M+1}$ com conjuntos cujos diâmetros são menores que $\min _{1 \leq i<j \leq M+1} d\left(x_{i}, x_{j}\right)$ são necessários no mínimo $M+1$ conjuntos.

Observação 2.3.7. $N\left(\mathfrak{U} \vee \varphi^{-1} \mathfrak{U} \vee \varphi^{-2} \mathfrak{U} \vee \ldots \vee \varphi^{-(n-1)} \mathfrak{U}\right) \leq N\left(\mathfrak{U} \vee \varphi^{-1} \mathfrak{U} \vee \varphi^{-2} \mathfrak{U} \vee\right.$ $\left.\ldots \vee \varphi^{-n} \mathfrak{U}\right)=N\left(\varphi^{-1} \mathfrak{U} \vee \varphi^{-2} \mathfrak{U} \vee \ldots \vee \varphi^{-n} \mathfrak{U}\right) \leq N\left(\mathfrak{U} \vee \varphi^{-1} \mathfrak{U} \vee \varphi^{-2} \mathfrak{U} \vee \ldots \vee \varphi^{-n+1} \mathfrak{U}\right)$ (Propriedades 2.1 .5 e 2.1.9). Portanto $N\left(\mathfrak{U} \vee \varphi^{-1} \mathfrak{U} \vee \varphi^{-2} \mathfrak{U} \vee \ldots \vee \varphi^{-n} \mathfrak{U}\right) \leq$ $N\left(\mathfrak{U} \vee \varphi^{-1} \mathfrak{U} \vee \varphi^{-2} \mathfrak{U} \vee \ldots \vee \varphi^{-n+1} \mathfrak{U}\right)$ 


\section{Capítulo 3}

\section{Entropia em Espaços Métricos e Razão de Crescimento}

\section{Exponencial}

Este capítulo tem como referência básica o artigo [23]. Apresentaremos uma definição de Entropia Topológica para espaços métricos, a definição da Razão de Crescimento Exponencial e relações importantes entre estes conceitos. Também serão estudadas algumas relações entre as duas definições de Entropia Topológica.

\subsection{Entropia em Espaços Métricos}

Em 1965 Adler, Konheim e McAndrew em [1] introduziram o conceito de entropia topológica para uma aplicação contínua $\varphi: X \rightarrow X$ de um espaço topológico compacto, que detalhamos no Capítulo 2.

Veremos neste capítulo uma formulação devido a Bowen [5] no caso que $X$ também é métrico.

Definição 3.1.1. Sejam $n \in \mathbb{N} e \varepsilon>0$. Um subconjunto $E \subset X$ é dito $(n, \varepsilon)$ bem distribuído para $\varphi$ se para todo ponto $x \in X$, existe um ponto $y \in E$ tal que $d\left(\varphi^{k}(x), \varphi^{k}(y)\right)<\varepsilon$ para $0 \leq k<n$. 
A compacidade de $X$ nos garante que $E$ pode ser escolhido finito; denotamos a cardinalidade mínima de um tal conjunto $E$ por $r(n, \varepsilon)$.

A definição de Bowen para entropia é:

Definição 3.1.2. A entropia topológica $h(\varphi)$ de $\varphi$ é definida como

$$
\lim _{\varepsilon \rightarrow 0} \lim _{n \rightarrow \infty} \sup \frac{1}{n} \log r(n, \varepsilon)
$$

Daremos agora uma definição dual de entropia topológica. Esta definição usará a idéia de conjuntos separados que é dual a noção de conjuntos bem distribuídos.

Definição 3.1.3. Sejam $n \in \mathbb{N} e \varepsilon>0$. Um subconjunto $E \subset X$ é dito $(n, \varepsilon)$ separado para $\varphi$ se $x, y \in E, x \neq y$ implica $d_{n}(x, y)>\varepsilon$ onde $d_{n}(x, y)=\max _{0 \leq i \leq n-1} d\left(\varphi^{i}(x), \varphi^{i}(y)\right)$; denotamos a cardinalidade máxima de qualquer subconjunto de $X(n, \varepsilon)$-separado por $s(n, \varepsilon)$.

Proposição 3.1.1. $r(n, \varepsilon) \leq s(n, \varepsilon) \leq r(n, \varepsilon / 2)$ e logo $s(n, \varepsilon)<\infty$.

Demonstração: Se $E$ é um subconjunto $(n, \varepsilon)$-separado de $X$ de cardinalidade máxima, então $E$ é um conjunto $(n, \varepsilon)$-bem distribuído para $X$. Portanto $r(n, \varepsilon) \leq s(n, \varepsilon)$. Para mostrar a outra desigualdade, suponha que $E$ é um subconjunto $(n, \varepsilon)$-separado de $X$ e $F$ é um conjunto $(n, \varepsilon / 2)$-bem distribuído para $X$. Defina $\phi: E \rightarrow F$ escolhendo para cada $x \in E$, algum ponto $\phi(x) \in F$ com $d(x, \phi(x)) \leq \varepsilon / 2$. Então $\phi$ é injetiva. De fato:

Seja $e_{1} \neq e_{2} \in E$. Provemos que $f_{1}=\phi\left(e_{1}\right) \neq \phi\left(e_{2}\right)=f_{2}$. 
Temos:

$$
\begin{array}{ll}
d\left(e_{1}, f_{1}\right)<\frac{\varepsilon}{2} & d\left(e_{2}, f_{2}\right)<\frac{\varepsilon}{2} \\
d\left(\phi\left(e_{1}\right), \phi\left(f_{1}\right)\right)<\frac{\varepsilon}{2} & d\left(\phi\left(e_{2}\right), \phi\left(f_{2}\right)\right)<\frac{\varepsilon}{2} \\
\cdots & \cdots \\
d\left(\phi^{n-1}\left(e_{1}\right), \phi^{n-1}\left(f_{1}\right)\right)<\frac{\varepsilon}{2} & d\left(\phi^{n-1}\left(e_{2}\right), \phi^{n-1}\left(f_{2}\right)\right)<\frac{\varepsilon}{2}
\end{array}
$$

Se $f_{1}=f_{2}$, temos para $k=1,2, \ldots,(n-1)$

$$
\begin{gathered}
d\left(\phi^{k}\left(e_{1}\right), \phi^{k}\left(e_{2}\right)\right) \leq d\left(\phi^{k}\left(e_{1}\right), \phi^{k}\left(f_{1}\right)\right)+d\left(\phi^{k}\left(e_{2}\right), \phi^{k}\left(f_{2}\right)\right)<\frac{\varepsilon}{2}+\frac{\varepsilon}{2}=\varepsilon . \\
\operatorname{Mas} \max _{k=1, \ldots, n} d\left(\phi^{k}\left(e_{1}\right), \phi^{k}\left(e_{2}\right)\right)>\varepsilon . \text { Absurdo! }
\end{gathered}
$$

Como $\phi$ é injetiva temos que a cardinalidade de $E$ não é maior que a de $F$. Logo, $s(n, \varepsilon) \leq r(n, \varepsilon / 2)$.

Observação 3.1.1. Se $\varepsilon_{1}<\varepsilon_{2}$, então $s\left(n, \varepsilon_{1}\right) \geq s\left(n, \varepsilon_{2}\right)$.

Definiçāo 3.1.4. A entropia topológica $h(\varphi)$ de $\varphi$ é definida neste caso como

$$
\lim _{\varepsilon \rightarrow 0} \lim _{n \rightarrow \infty} \sup \frac{1}{n} \log s(n, \varepsilon)
$$

Logo, $h(\varphi)$ pode ser definida usando conjuntos bem distribuídos ou conjuntos separados.

Bowen provou que $h(\varphi)$ não muda se a métrica sobre $X$ é substituída por uma métrica equivalente. De fato:

Teorema 3.1.1. [27] Quando X é compacto, a definição de Bowen (de entropia) coincide com a definição por cobertura aberta.

Demonstração: Seja $h^{*}(\varphi, \mathfrak{U})$ a entropia topológica de uma aplicação $\varphi: X \rightarrow X$ contínua com respeito a uma cobertura aberta $\mathfrak{U}$ e $h^{*}(\varphi)$ a entropia de $\varphi$, que ocorre na definição de entropia topológica por coberturas abertas. Seja $\mathfrak{U}=\left\{A_{1}, \ldots, A_{p}\right\}$ uma cobertura aberta de $X$ e $h(\varphi)$ a definição dada acima. 
Mostremos que $h^{*}(\varphi, \mathfrak{U}) \leq h(\varphi)$. Seja $\delta$ um número de Lebesgue para $\mathfrak{U}$. Seja $E$ um conjunto $(n, \delta / 2)$-bem distribuído para $X$ de cardinalidade mínima. Para $y \in E$ escolha $A_{i_{0}}(y), \ldots, A_{i_{n-1}}(y)$ em $\mathfrak{U}$ tal que $\overline{B_{\frac{\delta}{2}}\left(\varphi^{k}(y)\right)} \subset A_{i_{k}}(y)$. Seja $C(y)=A_{i_{0}}(y) \cap \varphi^{-1} A_{i_{1}}(y) \cap \ldots \cap \varphi^{-(n-1)} A_{i_{n-1}}(y)$, que é um membro de $\mathfrak{U} \vee \varphi^{-1} \mathfrak{U} \vee \ldots \vee \varphi^{-(n-1)} \mathfrak{U}$

Temos que $X=\bigcup_{y \in E} C(y)$, já que se $x \in X, \exists y \in E$ tal que $\max _{0 \leq i \leq n-1} d\left(\varphi^{i}(x), \varphi^{i}(y)\right) \leq \frac{\delta}{2}$. Logo, $x \in \varphi^{-k}\left(B_{\frac{\delta}{2}}\left(\varphi^{k}(y)\right)\right) \subset \varphi^{-k} A_{i_{b}}(y), 0 \leq k \leq n-1$; assim $x \in C(y)$.

Como $N\left(\mathfrak{U} \vee \varphi^{-1} \mathfrak{U} \vee \ldots \vee \varphi^{-(n-1)} \mathfrak{U}\right) \leq|E|=r(n, \delta / 2)$ temos que

$$
\begin{gathered}
\log N\left(\mathfrak{U} \vee \varphi^{-1} \mathfrak{U} \vee \ldots \vee \varphi^{-(n-1)} \mathfrak{U}\right) \leq \log r(n, \delta / 2) \\
\Rightarrow \lim _{n \rightarrow \infty} \log N\left(\mathfrak{U} \vee \varphi^{-1} \mathfrak{U} \vee \ldots \vee \varphi^{-(n-1)} \mathfrak{U}\right) \leq \lim _{n \rightarrow \infty} \log r(n, \delta / 2) \\
\Rightarrow \lim _{n \rightarrow \infty} \frac{1}{n} \log N\left(\mathfrak{U} \vee \varphi^{-1} \mathfrak{U} \vee \ldots \vee \varphi^{-(n-1)} \mathfrak{U}\right) \leq \lim _{n \rightarrow \infty} \frac{1}{n} \log r(n, \delta / 2) .
\end{gathered}
$$

Logo

$$
h^{*}(\varphi, \mathfrak{U}) \leq \lim _{n \rightarrow \infty} \sup \frac{1}{n} \log r(n, \delta / 2) \leq h(\varphi) .
$$

Portanto, $h^{*}(\varphi) \leq h(\varphi)$.

Para provar a recíproca, seja $\delta>0$.

Escolha uma cobertura aberta $\mathfrak{U}=\left\{A_{1}, \ldots, A_{p}\right\}$ de $X$ tal que $\operatorname{diam}\left(A_{i}\right)<\delta$ para todo $i$. Seja $F$ um subconjunto $(n, \delta)$-separado de $\mathrm{X}$ com cardinalidade máxima. Dois membros de $\mathrm{F}$ não podem pertencer ao mesmo elemento de $\mathfrak{U} \mathrm{V}$ $\varphi^{-1} \mathfrak{U} \vee \ldots \vee \varphi^{-(n-1)} \mathfrak{U}$ pois se $x, y \in \bigcap_{j=0}^{n-1} \varphi^{-j} A_{i_{j}}, \quad x, y \in F$, então $\max _{0 \leq j \leq n-1} d\left(\varphi^{j}(x), \varphi^{j}(y)\right)<\delta$ e $\operatorname{assim} x=y$.

Logo,

$$
\begin{aligned}
& N\left(\mathfrak{U} \vee \varphi^{-1} \mathfrak{U} \vee \ldots \vee \varphi^{-(n-1)} \mathfrak{U}\right) \geq|F|=s(n, \delta) . \\
\Rightarrow & \log N\left(\mathfrak{U} \vee \varphi^{-1} \mathfrak{U} \vee \ldots \vee \varphi^{-(n-1)} \mathfrak{U}\right) \geq \log s(n, \delta)
\end{aligned}
$$




$$
\begin{gathered}
\Rightarrow \lim _{n \rightarrow \infty} \log N\left(\mathfrak{U} \vee \varphi^{-1} \mathfrak{U} \vee \ldots \vee \varphi^{-(n-1)} \mathfrak{U}\right) \geq \lim _{n \rightarrow \infty} \log s(n, \delta) \\
\Rightarrow \lim _{n \rightarrow \infty} \frac{1}{n} \log N\left(\mathfrak{U} \vee \varphi^{-1} \mathfrak{U} \vee \ldots \vee \varphi^{-(n-1)} \mathfrak{U}\right) \geq \lim _{n \rightarrow \infty} \frac{1}{n} \log s(n, \delta) .
\end{gathered}
$$

Portanto, $h^{*}(\varphi) \geq h^{*}(\varphi, \mathfrak{U}) \geq \lim _{n \rightarrow \infty} \frac{1}{n} \log s(n, \delta)$.

Fazendo $\delta \rightarrow 0$ temos $h^{*}(\varphi) \geq h(\varphi)$.

Como, quando $X$ é compacto, a definição de entropia topológica de Bowen coincide com a definição por coberturas abertas, isto significa que, neste caso, a entropia topológica $h(\varphi)$ é independente da métrica, ou seja, $h(\varphi)$ não muda se a métrica sobre $X$ é substituída por uma métrica equivalente.

Podemos ver $h(\varphi)$ como uma medida da complexidade de $\varphi$. É possível verificar que:

(1) A entropia topológica de qualquer aplicação periódica é zero,

(2) A entropia topológica de qualquer homeomorfismo de superfície pseudoAnosov é positiva.

Vejamos alguns exemplos do cálculo de entropia com esta definição:

Exemplo 3.1.1. Consideremos $X=[0,1], \varphi=i d$

$$
i d:[0,1] \rightarrow[0,1]
$$

temos que $h(\varphi)=0$.

Demonstração: Sejam $\varepsilon=\frac{1}{m}, \varepsilon>0$ e $n \in \mathbb{N}$. Tomemos $E \subset X$ tal que

$$
E=\left\{\frac{1}{m+1}, \frac{2}{m+1}, \ldots, \frac{m}{m+1}\right\}
$$

Temos que para todo $x \in X=[0,1]$ existe um ponto $y \in E$ tal que 


$$
d(i d(x), i d(y))=d(x, y)<\varepsilon=\frac{1}{m}
$$

pois $d\left(\frac{i}{m+1}, \frac{i+1}{m+1}\right)=\frac{1}{m+1}<\frac{1}{m}=\varepsilon$.

Assim temos:

Se $x \in E$, então $x$ é da forma $\frac{i}{m+1} \operatorname{com} 1 \leq i \leq m$. Basta tomar $y=x$ e teremos $d(x, y)=0<\varepsilon$.

Se $x \in X-E$, então $x \in\left(\frac{i}{m+1}, \frac{i+1}{m+1}\right)$ com $1 \leq i \leq m$. Basta tomar $y=\frac{i}{m+1}$ e teremos $d(x, y)<\varepsilon$.

Portanto, $E$ é $(n, \varepsilon)$-bem distribuído. Temos também que $r(n, \varepsilon) \leq m$ que é a cardinalidade de $E$. Então

$$
\begin{gathered}
\lim _{\varepsilon \rightarrow 0}\left(\lim _{n \rightarrow \infty} \sup \frac{1}{n} \log r(n, \varepsilon)\right) \leq \lim _{\varepsilon \rightarrow 0}\left(\lim _{n \rightarrow \infty} \sup \frac{1}{n} \log m\right)=\lim _{m \rightarrow \infty}\left(\lim _{n \rightarrow \infty} \sup \frac{1}{n} \log m\right) \\
=\lim _{m \rightarrow \infty}\left(\log m . \lim _{n \rightarrow \infty} \sup \frac{1}{n}\right)=\lim _{m \rightarrow \infty}\left(\log m \cdot \lim _{n \rightarrow \infty} \frac{1}{n}\right) \\
=\lim _{m \rightarrow \infty}(\log m \cdot 0)=\lim _{m \rightarrow \infty} 0=0 .
\end{gathered}
$$

Portanto a entropia topológica $h(\varphi)=0$.

Exemplo 3.1.2. Seja $\varphi:[0,1] \rightarrow[0,1]$ definida por $\varphi(x)=C_{0}$. Temos que $h(\varphi)=0$.

Demonstração: Sejam $\varepsilon=\frac{1}{m}, \varepsilon>0$ e $n \in \mathbb{N}$. Tomemos $E$ como no exempilo. anterior.

Temos que para todo $x \in X=[0,1]$, existe um ponto $y \in E$ tal que:

$$
d\left(\varphi^{k}(x), \varphi^{k}(y)\right)=d\left(C_{0}, C_{0}\right)=0<\varepsilon=\frac{1}{m} .
$$

Portanto $E$ é $(n, \varepsilon)$-bem distribuído. Temos também que $r(n, \varepsilon) \leq m$, onde $m$ é a cardinalidade de $E$. Então, como anteriormente $h(\varphi)=0$. 
Entropia topológica tem muitas propriedades interessantes, entre elas, a invariância de $h(\varphi)$ em relação a conjugação topológica (isto é, conjugação por um homeomorfismo) descrita pelo próximo lema. Os dois lemas a seguir estão em [1] e foram detalhados no capítulo 2 .

Lema 3.1.1. Seja $\varphi_{1}: X_{1} \rightarrow X_{1}$ e $\varphi_{2}: X_{2} \rightarrow X_{2}$ aplicações contínuas de espaços métricos compactos. Se existe $g: X_{1} \rightarrow X_{2}$ contínua e sobrejetora tal que $g \varphi_{1}=\varphi_{2} g$, então $h\left(\varphi_{1}\right) \geq h\left(\varphi_{2}\right)$. Em particular, se $g$ é um homeomorfismo, então $h\left(\varphi_{1}\right)=h\left(\varphi_{2}\right)$.

Lema 3.1.2. Se $\varphi: X \rightarrow X$ é um homeomorfismo de um espaço compacto, então $h\left(\varphi^{k}\right)=|k| h(\varphi)$ para qualquer inteiro $k$. Em particular, $h\left(\varphi^{-1}\right)=h(\varphi)$.

Observemos que por este lema, no Exemplo 3.1.1, a entropia $h(I d)$ tem que ser zero, pois, se tivéssemos $h(I d) \neq 0$ então pelo lema acima teríamos $h\left(I d^{k}\right)=$ $|k| h(I d)$, mas $I d^{k}=I d$, então temos $h(I d)=|k| h(I d), \forall k$ que só é válido se $h(I d)=0$.

\subsection{Razão de Crescimento Exponencial}

Bowen introduziu em [4] a razão de crescimento exponencial de endomorfismo de grupo para estimar entropia topológica. Uma versão logarítmica descrita por Fathi e Shub em [9] é revista aqui.

Seja $G$ grupo com um conjunto finito de geradores $g_{1}, \ldots, g_{n}$, e seja $\alpha: G \rightarrow G$ um endomorfismo. A razão de crescimento exponencial $E G R(\alpha)$ é definida como

$$
\sup _{g \in G} \lim _{k \rightarrow \infty} \sup \frac{1}{k} \log \left|\alpha^{k}(g)\right|
$$


onde $|g|$ denota o comprimento da menor palavra em $g_{1}^{ \pm 1}, \ldots, g_{n}^{ \pm 1}$ representando $g$.

Observemos que o supremo na definição pode ser substituído pelo máximo sobre os geradores $g_{i}, 1 \leq i \leq n$.

De fato:

Sabemos que

$$
\sup _{g_{i}} \lim _{k \rightarrow \infty} \sup \frac{1}{k} \log \left|\alpha^{k}\left(g_{i}\right)\right| \leq \sup _{g \in G} \lim _{k \rightarrow \infty} \sup \frac{1}{k} \log \left|\alpha^{k}(g)\right| .
$$

Ou seja,

$$
\max _{g_{i}} \lim _{k \rightarrow \infty} \sup \frac{1}{k} \log \left|\alpha^{k}\left(g_{i}\right)\right| \leq \sup _{g \in G} \lim _{k \rightarrow \infty} \sup \frac{1}{k} \log \left|\alpha^{k}(g)\right|,
$$

já que $\sup _{g_{i}}=\max _{g_{i}}$ pois estamos considerando um número finito de geradores.

Mostremos que:

$$
\lim _{k \rightarrow \infty} \sup \frac{1}{k} \log \left|\alpha^{k}(g)\right| \leq \max _{g_{i}} \lim _{k \rightarrow \infty} \sup \frac{1}{k} \log \left|\alpha^{k}\left(g_{i}\right)\right| .
$$

Observe que existe índice $i_{0}$, tal que

$$
\max _{g_{i}} \lim _{k \rightarrow \infty} \sup \frac{1}{k} \log \left|\alpha^{k}\left(g_{i}\right)\right|=\lim _{k \rightarrow \infty} \sup \frac{1}{k} \log \left|\alpha^{k}\left(g_{i_{0}}\right)\right| \text {, para algum } g_{i_{0}} \text {. }
$$

Seja $g \in G \operatorname{com}|g|=r$, então $g=g_{i_{1}}^{\varepsilon_{1}} \cdot g_{i_{2}}^{\varepsilon_{2}} \ldots g_{i_{r}}^{\varepsilon_{r}}$, onde $\varepsilon_{i} \in\{-1,1\}$.

Temos que:

$\lim _{k \rightarrow \infty} \sup \frac{1}{k} \log \left|\alpha^{k}(g)\right|=\lim _{k \rightarrow \infty} \sup \frac{1}{k} \log \left|\alpha^{k}\left(g_{i_{1}}^{\varepsilon_{1}} \cdot g_{i_{2}}^{\varepsilon_{2}} \ldots g_{i_{r}}^{\varepsilon_{r}}\right)\right|=$

$=\lim _{k \rightarrow \infty} \sup \frac{1}{k} \log \left|\alpha^{k}\left(g_{i_{1}}^{\varepsilon_{1}}\right) \cdot \alpha^{k}\left(g_{i_{2}}^{\varepsilon_{2}}\right) \ldots \alpha^{k}\left(g_{i_{r}}^{\varepsilon_{r}}\right)\right| \leq \lim _{k \rightarrow \infty} \sup \frac{1}{k} \log r\left|\alpha^{k}\left(g_{i_{0}}\right)\right|=$

$=\lim _{k \rightarrow \infty} \sup \frac{1}{k}\left[\log r+\log \left|\alpha^{k}\left(g_{i_{0}}\right)\right|\right]=\lim _{k \rightarrow \infty} \sup \frac{1}{k} \log \left|\alpha^{k}\left(g_{i_{0}}\right)\right|=$

$=\max _{g_{i}} \lim _{k \rightarrow \infty} \sup \frac{1}{k} \log \left|\alpha^{k}\left(g_{i}\right)\right|$,

onde a última igualdade é devido a observação. 
Também, $E G R(\alpha)$ é finito, independente do conjunto de geradores, e não muda se $\alpha$ é composto com um automorfismo interno de $G$.

De fato: Vejamos inicialmente que $E G R(\alpha)$ é finito, independente do conjunto dos geradores.

Dado um grupo $G$ com apresentação $\left|g_{1}, g_{2}, \ldots, g_{n}: r_{1}, \ldots, r_{s}\right|$ e $g \in G$, sejam $G_{1}=\left\{g_{1}, \ldots, g_{r}\right\}$ e $G_{1}^{\prime}=\left\{g_{1}^{\prime}, \ldots, g_{r}^{\prime}\right\}$ dois conjuntos de geradores de $G$, então pelo que observamos anteriormente temos que

$$
\sup _{g \in G} \lim _{k \rightarrow \infty} \frac{1}{k} \log \left|\alpha^{k}(g)\right|=\max _{g \in G G_{1}} \lim _{k \rightarrow \infty} \frac{1}{k} \log \left|\alpha^{k}\left(g_{i}\right)\right|,
$$

mas também temos

$$
\sup _{g \in G} \lim _{k \rightarrow \infty} \frac{1}{k} \log \left|\alpha^{k}(g)\right|=\max _{g_{i}^{\prime} \in G_{1}^{\prime}} \lim _{k \rightarrow \infty} \frac{1}{k} \log \left|\alpha^{k}\left(g_{i}^{\prime}\right)\right| .
$$

Portanto $E G R(\varphi)$ não depende do conjunto dos geradores, e este valor é finito, pois estamos trabalhando com um número finito de geradores.

Mostremos agora que $E G R(\alpha)$ não muda se $\alpha$ é composta com um automorfismo interno de G.

Se $\alpha: G \rightarrow G$ é um endomorfismo e $g \in G$, defina $g \alpha g^{-1}: G \rightarrow G$ por $\left[g \alpha g^{-1}\right](x)=g \alpha(x) g^{-1}$. Mostremos que $E G R(\alpha)=E G R\left(g \alpha g^{-1}\right)$. (Atenção: $\left.\left(g \alpha g^{-1}\right)^{n} \neq\left(g \alpha^{n} g^{-1}\right)\right)$

Se $x \in G$ temos:

$$
\begin{aligned}
& \left(g \alpha g^{-1}\right)^{n}(x)=\left(g \alpha g^{-1}\right)^{n-1}\left(g \alpha(x) g^{-1}\right)=\left(g \alpha g^{-1}\right)^{n-2}\left[g \alpha\left(\alpha(x) g^{-1}\right) g^{-1}\right]= \\
= & \left(g \alpha g^{-1}\right)^{n-2}\left[g\left(\alpha(g) \alpha^{2}(x) \alpha\left(g^{-1}\right)\right) g^{-1}\right]=\left(g \alpha g^{-1}\right)^{n-3}\left[g \alpha\left(g \alpha(g) \alpha^{2}(x) \alpha\left(g^{-1}\right) g^{-1}\right) g^{-1}\right]= \\
= & \left(g \alpha g^{-1}\right)^{n-3}\left[g\left(\alpha(g) \alpha^{2}(g) \alpha^{3}(x) \alpha^{2}\left(g^{-1}\right) \alpha\left(g^{-1}\right)\right) g^{-1}\right]= \\
= & \left(g \alpha g^{-1}\right)^{n-4}\left[g \alpha\left(g \alpha(g) \alpha^{2}(g) \alpha^{3}(x) \alpha^{2}\left(g^{-1}\right) \alpha\left(g^{-1}\right) g^{-1}\right) g^{-1}\right]= \\
= & \left(g \alpha g^{-1}\right)^{n-4}\left[g \alpha(g) \alpha^{2}(g) \alpha^{3}(g) \alpha^{4}(x) \alpha^{3}\left(g^{-1}\right) \alpha^{2}\left(g^{-1}\right) \alpha\left(g^{-1}\right) g^{-1} g^{-1}\right]= \\
= & \ldots=g \alpha(g) \alpha^{2}(g) \ldots \alpha^{n-1}(g) \alpha^{n}(x) \alpha^{n-1}\left(g^{-1}\right) \ldots \alpha^{2}\left(g^{-1}\right) \alpha\left(g^{-1}\right) g^{-1} .
\end{aligned}
$$


(a) Suponhamos que $\alpha^{n_{0}}(g)=e \Rightarrow \alpha^{n_{0}}\left(g^{-1}\right)=\left[\alpha^{n_{0}}(g)\right]^{-1}=e$.

Então para $N \gg n_{0}$, temos que $\left(g \alpha g^{-1}\right)^{N}(x)=g \alpha(g) \alpha^{2}(g) \ldots \alpha^{n_{0}-1}(g) \alpha^{n}(x) \alpha^{n_{0}-1}\left(g^{-1}\right) \ldots \alpha^{2}\left(g^{-1}\right) \alpha\left(g^{-1}\right) g^{-1}$.

Logo, $\left|\left(g \alpha g^{-1}\right)(x)\right|^{N}=$ constante $+\left|\alpha^{n}(x)\right|$.

Portanto pela Proposição 1.0.7 (i): $\lim \sup \frac{1}{n} \log \left|\left(g \alpha g^{-1}\right)^{n}(x)\right| \leq \lim \sup \frac{1}{n} \log [c t+$ $\left.\left|\alpha^{n}(x)\right|\right]=\max \left\{\lim \sup \frac{1}{n} \log (c t), \lim \sup \frac{1}{n} \log \left|\alpha^{n}(x)\right|\right\}=\lim \sup \frac{1}{n} \log \left|\alpha^{n}(x)\right|$.

(b) Se $\alpha^{n}(g) \neq e$ para cada $n \geq 1$, temos $\left|\alpha^{n}(g)\right| \geq 1$ para cada $n \geq 1$. Logo, $\lim \sup \frac{1}{n} \log \left|\alpha^{n}(g)\right| \geq 0$.

Temos que:

$\left|\left(g \alpha g^{-1}\right)(x)\right|^{N}=\left|g \alpha(g) \alpha^{2}(g) \ldots \alpha^{N-1}(g) \alpha^{N}(x) \alpha^{N-1}\left(g^{-1}\right) \ldots \alpha^{2}\left(g^{-1}\right) \alpha\left(g^{-1}\right) g^{-1}\right| \leq$ $\leq\left|g \alpha(g) \alpha^{2}(g) \ldots \alpha^{N-1}(g)\right|+\left|\alpha^{N}(x)\right|+\left|\alpha^{N-1}\left(g^{-1}\right) \ldots \alpha^{2}\left(g^{-1}\right) \alpha\left(g^{-1}\right) g^{-1}\right|$.

Observemos que $\left|g \alpha(g) \alpha^{2}(g) \ldots \alpha^{N-1}(g)\right| \leq|g|+|\alpha(g)|+\ldots+\left|\alpha^{N-1}(g)\right|$.

Assim, pela Proposição 1.0.7 (i) e (iii), temos que:

$\lim _{N \rightarrow \infty} \sup \frac{1}{N} \log \left|\alpha^{N-1}(g)\right| \leq \lim _{N \rightarrow \infty} \sup \frac{1}{N} \log \left(|g|+|\alpha(g)|+\ldots+\left|\alpha^{N-1}(g)\right|\right)$ $\leq \max \left\{0, \lim _{N \rightarrow \infty} \sup \frac{1}{N} \log \left|\alpha^{N-1}(g)\right|\right\}$.

Mas sabemos que limsup $\frac{1}{n} \log \left|\alpha^{n}(g)\right| \geq 0$. Logo, $\left.\max \left\{0, \lim _{N \rightarrow \infty} \sup \frac{1}{N} \log \left|\alpha^{N-1}(g)\right|\right\}=\lim _{N \rightarrow \infty} \sup \frac{1}{N} \log \left|\alpha^{N-1}(g)\right|\right\}$.

Além disso, $\left|g \alpha(g) \alpha^{2}(g) \ldots \alpha^{N-1}(g)\right|=\left|\alpha^{N-1}\left(g^{-1}\right) \ldots \alpha^{2}\left(g^{-1}\right) \alpha\left(g^{-1}\right) g^{-1}\right|$.

Portanto, $\left|\left(g \alpha g^{-1}\right)(x)\right|^{N} \leq 2\left|g \alpha(g) \alpha^{2}(g) \ldots \alpha^{N-1}(g)\right|+\left|\alpha^{N}(x)\right|$.

Logo, também pela Proposição 1.0.7,

$\lim _{N \rightarrow \infty} \sup \frac{1}{n} \log \left|\left(g \alpha g^{-1}\right)^{N}(x)\right| \leq \lim _{N \rightarrow \infty} \sup \frac{1}{n} \log \left(2\left|g \alpha(g) \alpha^{2}(g) \ldots \alpha^{N-1}(g)\right|+\left|\alpha^{N}(x)\right|\right)$ $\leq \lim _{N \rightarrow \infty} \sup \frac{1}{n} \log \left[2\left|\alpha^{N-1}(g)\right|+\left|\alpha^{N}(x)\right|\right]$ $\leq \max \left\{\lim \sup \frac{1}{n} \log \left|\alpha^{N}(g)\right|, \lim \sup \frac{1}{n} \log \left|\alpha^{N}(x)\right|\right\}$.

Assim temos que $E G R\left(g \alpha g^{-1}\right) \leq E G R(\alpha)$ e por simetria, temos $E G R\left(g \alpha g^{-1}\right)=$ $E G R(\alpha)$, como queríamos. 
Bowen, em [5], provou que se $\varphi: M \rightarrow M$ é qualquer aplicação contínua em uma variedade compacta, então a razão de crescimento exponencial do endomorfismo induzido $\varphi_{\sharp}: \pi_{1}(M) \rightarrow \pi_{1}(M)$ é um limitante inferior da entropia topológica $h(\varphi)$.

É tentador olhar razão de crescimento exponencial como entropia na categoria dos grupos finitamente gerados já que muitas propriedades da entropia topológica valem para razão de crescimento exponencial, como demonstra o lema seguinte análogo ao Lema 3.1.1.

Lema 3.2.1. [9] Seja $\alpha_{1}: G_{1} \rightarrow G_{1}$ e $\alpha_{2}: G_{2} \rightarrow G_{2}$ endomorfismos de grupos finitamente gerados. Se existe um epimorfismo $\beta: G_{1} \rightarrow G_{2}$ tal que $\beta \alpha_{1}=\alpha_{2} \beta$, então $E G R\left(\alpha_{1}\right) \geq E G R\left(\alpha_{2}\right)$. Em particular, se $\beta$ é um isomorfismo, então $E G R\left(\alpha_{1}\right)=E G R\left(\alpha_{2}\right)$.

Contudo nem todas as propriedades de entropia topológica valem para razão de crescimento exponencial. Por exemplo, se $\alpha: G \rightarrow G$ é um automorfismo de um grupo finitamente gerado, então $E G R(\alpha)$ não precisa ser igual a $E G R\left(\alpha^{-1}\right)$.

Observe outra diferença significante entre entropia topológica e razão de crescimento exponencial. Se $\varphi: X \rightarrow X$ é uma aplicação contínua de um espaço compacto e $A \subset X$ é um subespaço fechado de $\mathrm{X}$ que é $\varphi$-invariante (isto é, $\varphi(A) \subset A$ ), então $h\left(\left.\varphi\right|_{A}\right) \leq h(\varphi)$ [1] (Teorema 4). Por outro lado, se $\alpha: G \rightarrow G$ é um endomorfismo de um grupo finitamente gerado e $H \subset G$ é um subgrupo $\alpha$-invariante finitamente gerado, então $E G R\left(\left.\alpha\right|_{H}\right)$ pode exceder $E G R(\alpha)$ (ver [22](p.219).

De fato:

Mergulhe $\mathrm{G}$ em $G^{*}=<G, t \mid t g t^{-1}=\alpha(g), g \in G>$ e estenda $\alpha$ a um automorfismo $\alpha^{*}: G^{*} \rightarrow G^{*}, t \rightarrow t$.

Como $\left(\alpha^{*}\right)^{k}(g)=\alpha^{k}(g)=t^{k} g t^{-k}$ para cada $g \in G$, então $E G R\left(\alpha^{*}\right)=0$.

Agora se $\alpha: G \rightarrow G$ é um endomorfismo de grupo livre e $H \subset G$ é um subgrupo finitamente gerado tal que $\alpha(H) \subset H$, então $E G R(\alpha) \geq E G R\left(\left.\alpha\right|_{H}\right)$. 


\section{Capítulo 4}

\section{Entropia de Nós}

Neste capítulo estaremos trabalhando na categoria de variedades diferenciáveis $\left(C^{\infty}\right)$. Um $n$-nó é uma $n$-esfera $K$ mergulhada numa esfera homológica $\Sigma$ de dimensão $n+2$. Dois $n$-nós $K_{1} \subset \Sigma_{1}$ e $K_{2} \subset \Sigma_{2}$ são equivalentes se existe um difeomorfismo $\varphi: \Sigma_{1} \rightarrow \Sigma_{2}$ tal que $\varphi\left(K_{1}\right)=K_{2}$. Dois $n$-nós equivalentes são vistos como o mesmo. Um $n$-nó $K \subset \Sigma$ é trivial se ele é o bordo de uma $(n+1)$-bola em $\Sigma$.

$O$ exterior de um $n$-nó $K \subset \Sigma$ é o fecho $X(K)$ de $\Sigma$ menos uma vizinhança tubular de $K$. Vemos o bordo $\partial X(K)$ como $K \times S^{1}$. Qualquer curva fechada simples $p \times S^{1}$ em $\partial X(K)$ é chamado um meridiano de $K$. O $n$-nó $K$ é fibrado se a projeção $K \times S^{1} \rightarrow S^{1}$ se estende para uma fibração localmente trivial $\phi: X(K) \rightarrow S^{1}$. Chamamos $\phi$ uma fibração de $K$. Neste caso, para cada $\theta \in S^{1}$ a pré-imagem $\phi^{-1}(\theta)$ é uma cópia de alguma $(n+1)$-variedade compacta, conexa $F$ com $\partial F=K$ chamada a fibra de $\phi$, e $X(K)$ é difeomorfo a um "mapping torus" $M_{\varphi}=F \times[0,1] /(x, 0) \sim(\varphi(x), 1)$, onde $\varphi: F \rightarrow F$ é um difeomorfismo chamado uma monodromia de $\phi$. Qualquer monodromia de $\phi$ pode ser descrita como em [10] por:

(1) selecionando um fluxo em $X(K)$ que é transversal a cada fibra de $\phi$;

(2) identificando $\phi^{-1}(0)$ com $F$, e para $x \in \phi^{-1}(0)$ seguindo a linha do fluxo através de $x$ na direção que corresponde a valores decrescentes de $\theta \in S^{1}$ até 
chegar à um ponto, definido por $\varphi(x)$, em $\phi^{-1}(1)=\phi^{-1}(0)$.

A coleção de todas as monodromias de $\phi$ é a classe de isotopia de $\varphi$.

Seja $K \subset \Sigma$ um $n$-nó fibrado, e assuma que $\varphi$ é uma monodromia de alguma fibração de $K$. Não é difícil obter a entropia topológica $h(\varphi)$ arbitrariamente grande modificando $\varphi$ por isotopia. Por outro lado, a não-negatividade da entropia topológica nos permite fazer a seguinte definição:

Definição 4.0.1. Se $K$ é um n-nó fibrado, então a entropia de $K$, denotada por $h_{K}$, é $\inf \{h(\varphi) \mid \varphi$ é monodromia de alguma fibraçäo de $K\}$.

Por exemplo: Consideremos um nó clássico, isto é, $S^{1} \subset S^{3}$.

Seja $K=\operatorname{Im}\left(S^{1}\right) \subset S^{3}, X(K)=\overline{S^{3}-V(K)}$, onde $V(K)=$ vizinhança tubular de $K$ e $\partial X(K)=K \times S^{1}$.

Fixe um meridiano $p \times S^{1}$ em $\partial X(K)$.

Suponha que o nó $K$ é fibrado e seja $\phi: X(K) \rightarrow S^{1}$ uma fibração.

Para cada ponto $\theta \in S^{1}, \phi^{-1}(\theta)$ é uma cópia de alguma superfície compacta, conexa $F$ chamada fibra de $\phi$.

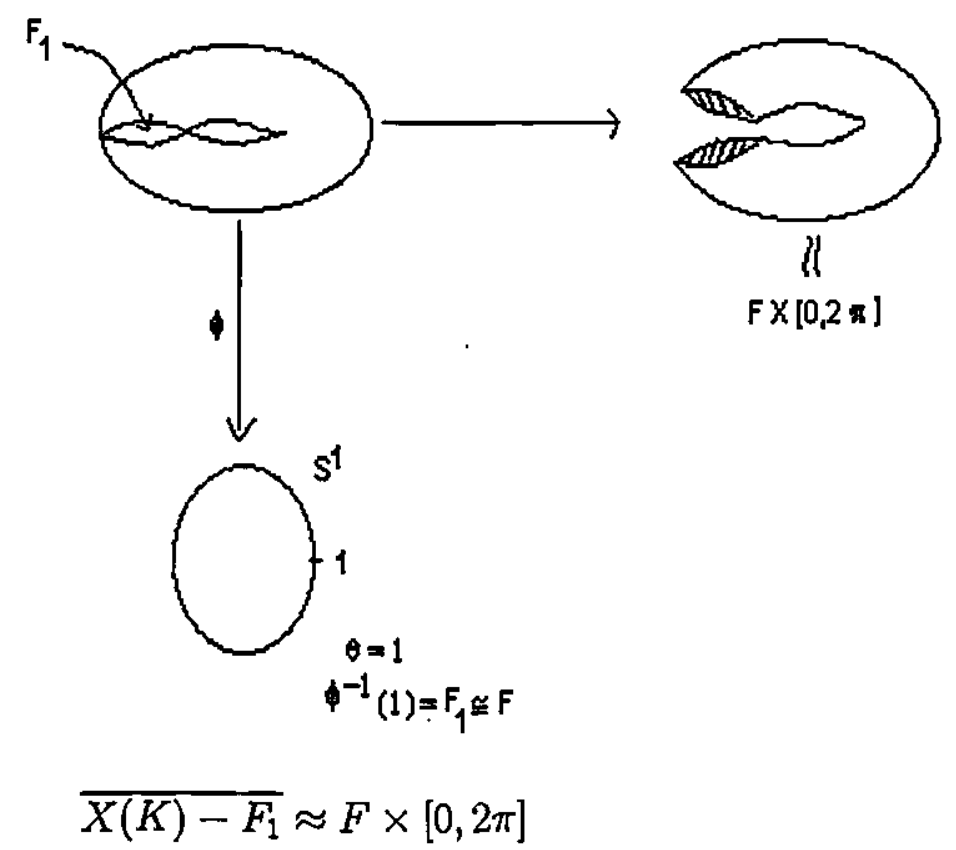


Para se obter $X(K)$ novamente de $F \times[0,2 \pi]$ considere a monodromia $\varphi: F \rightarrow F$ dando origem à $\varphi: F \times\{0\} \rightarrow F \times\{2 \pi\}$, então

$$
\frac{F \times[0,2 \pi]}{\varphi} \approx X(K) .
$$

Então temos o seguinte esquema:

$K$, nó fibrado $\longrightarrow \varphi: F \rightarrow F$, monodromia $\longrightarrow h(\varphi)$, entropia ou seja, a partir de um nó fibrado, obtemos monodromias de fibras e calculamos então a entropia dessas monodromias.

Tomando-se o ínfimo dessas entropias, obtemos a entropia do nó fibrado $K$.

É fácil ver que $h_{K}$ é um invariante do nó, isto é, ela depende da classe de $K$ e se $K_{1} \sim K_{2}$ temos $h\left(K_{1}\right)=h\left(K_{2}\right)$.

Para qualquer 1-nó fibrado $K$, um teorema de Blank e Laudenbach [3] nos permite calcular $h_{K}$ considerando uma única fibração de $K$. Pelo teorema, se $\phi_{1}$ e $\phi_{2}$ são quaisquer fibrações de $K$, então $\phi_{2}$ é isotópica a $\phi_{1}$ ou $-\phi_{1}$. Consequentemente, se $\varphi_{1}: F_{1} \rightarrow F_{1}$ e $\varphi_{2}: F_{2} \rightarrow F_{2}$ são monodromias das respectivas fibraçōes $\phi_{1}$ e $\phi_{2}$ de $K$, então ou $g \varphi_{1}=\varphi_{2}^{\prime} g$ ou $g \varphi_{1}^{-1}=\varphi_{2}^{\prime} g$ para alguma monodromia $\varphi_{2}^{\prime}$ de $\phi_{2}$ e difeomorfismo $g: F_{1} \rightarrow F_{2}$. Pelos Lemas 3.1.1 e 3.1.2 as entropias topológicas $h\left(\varphi_{1}\right)$ e $h\left(\varphi_{2}^{\prime}\right)$ são iguais. Segue que $\inf \left\{h\left(\varphi_{1}\right) \mid \varphi_{1}\right.$ é uma monodromia de $\left.\phi_{1}\right\}$ e inf $\left\{h\left(\varphi_{2}\right) \mid \varphi_{2}\right.$ é uma monodromia de $\left.\phi_{2}\right\}$ são os mesmos.

Se $K$ é um $n$-nó fibrado com $n>1$, então fibras de diferentes fibrações de $K$ não precisam ser difeomorfas.

Lema 4.0.2. Suponhamos que $\varphi_{1}: F_{1} \rightarrow F_{1}$ e $\varphi_{2}: F_{2} \rightarrow F_{2}$ são monodromias de fibrações dos respectivos n-nós $K_{1}$ e $K_{2}$. Se o exterior $X\left(K_{1}\right)$ e $X\left(K_{2}\right)$ são equivalentes homotópicos, então existe uma equivalência homotópica $g: F_{1} \rightarrow F_{2}$ tal que ou $g \varphi_{1}$ ou $g \varphi_{1}^{-1}$ é homotópica a $\varphi_{2} g$. 
Observação 4.0.1. Uma aplicação contínua $\varphi: X \rightarrow Y$ chama-se uma equivalência homotópica quando existe $g: Y \rightarrow X$ contínua tal que $g \circ \varphi \simeq i d_{X} e$ $\varphi \circ g \simeq i d_{Y}$. Neste caso dizemos que $X$ e $Y$ tem o mesmo tipo de homotopia.

Embora seja difícil calcular $h_{K}$ para um $n$-nó fibrado arbitrário $K$, há uma boa razão para se estudar este invariante $h_{K}$, pois o mesmo é extremamente sensível, isto é, detecta muitas complicações.

Temos também que a razão de crescimento exponencial vista no Capítulo 3 pode ser usada para estimar a entropia $h_{K}$ de um $n$-nó $K$.

Assuma que $K$ é qualquer $n$-nó, orientado, e denote seu grupo $\pi_{1}(X(K))$ pelo símbolo $G_{K}$. Seja $t \in G_{K}$ representado por algum meridiano de $K$ de preferência com orientação induzida por $K$.

Então a conjugação $x \rightarrow t x t^{-1}, x \in G_{k}$, induz um automorfismo $\mu_{t}$, do subgrupo comutador $G_{K}^{\prime}$. Se $G_{K}^{\prime}$ é finitamente gerado (como é o caso de nós fibrados), então $E G R\left(\mu_{t}\right)$ está definido. Em [22] temos algumas das propriedades acima mencionadas da razão de crescimento exponencial para se provar que $E G R\left(\mu_{t}\right)$ é um invariante $\gamma_{K}$ de $n$-nós orientados $K$. Se, além disso, $K$ é fibrado com monodromia $\varphi: F \rightarrow F$, entāo podemos identificar $G_{K}^{\prime} \operatorname{com} \pi_{1}(F)$, e a menos de composição com um automorfismo interno de $G_{K}^{\prime}$ podemos identificar $\mu_{t} \operatorname{com} \varphi_{\sharp}$ ou $\varphi_{\sharp}^{-1}$ (dependendo da direção do fluxo usada para definir $\varphi$ ). Pelo Lema 3.1.2 juntamente com o Teorema de Bowen, Gromov, Manning e Shub, $\gamma_{K} \leq h(\varphi)$. Como $\varphi$ é uma monodromia arbitrária de $K$, o invariante $\gamma_{K}$ é um limitante inferior de $h_{K}$. Além disso, se revertermos a orientação de $K$, assim obtendo o $n$-nó orientado que denotamos por $r K$, então $\gamma_{r K} \leq h_{K}$ pelo mesmo argumento. Logo temos os seguintes teoremas:

Teorema 4.0.1. Se K é um n-nó fibrado orientado, então $\max \left\{\gamma_{K}, \gamma_{r K}\right\} \leq h_{K}$.

Teorema 4.0.2. Se $K$ é qualquer 1-nó fibrado, então $h_{K}=\gamma_{K}$. 
Quando um nó não é fibrado não podemos usar os resultados anteriores. No entanto, existe uma forma de contornar esta situação.

Seja $K$ um nó com exterior $X$. Em [15] Kakimizu provou que $X$ contém uma subvariedade compacta, conexa de codimensão $0, X_{0}$ tal que cada componente do bordo $\partial X_{0}$ é incompressivel em $X$, e tal que :

(i) $\partial X=\left(K \times S^{1}\right) \subset \partial X_{0}$,

(ii) a projeção $K \times S^{1} \rightarrow S^{1}$ se estende à uma fibração $\phi: X_{0} \rightarrow S^{1}$,

(iii) $X_{0}$ é maximal e única (a menos de isotopia em $X$ ) com respeito às condições (i) e (ii).

Da mesma forma que em [15], chamaremos $X_{0}$ a subvariedade maximal fibrada de $K$. Qualquer fibração $\phi$ como na condição (ii) será chamada uma fibração maximal de $K$. Kakimizu provou em [15] que uma fibra $S_{0}$ de $\phi$ pode ser realizada como uma subsuperfície de alguma superfície de Seifert incompressível $S$ de $K$. Ele também mostrou que

$$
\pi_{1}\left(S_{0}, *\right)=\bigcap_{z \in Z} t^{k} \pi_{1}(S, *) t^{-1}
$$

onde $t$ é a classe de um meridiano $* \times S^{1} \subset \partial X$. Note em particular que como $S_{0}$ é compacta, $\pi_{1}\left(S_{0}, *\right)$ é finitamente gerado (livre). Também, o automorfismo $\mu$ de $G_{k}^{\prime}$ definido por $\mu_{t}(x)=t x t^{-1}$ (ver Capítulo 3 ) se restringe a um automorfismo de $\pi_{1}\left(S_{0}, *\right)$.

Exemplo 4.0.1. Um nó $K$ é fibrado se e somente se $X_{0}=X$.

Exemplo 4.0.2. Se $K$ não é fibrado $e X$ é atoroidal (isto é, qualquer toro incompressivel è $X$ é paralelo ao bordo), então $X_{0}=($ colarinho de $\partial S) \times S^{1}$. Neste caso, a subvariedade maximal fibrada $X_{0}$ é dita trivial . 
Se $\phi: X_{0} \rightarrow S^{1}$ é uma fibração maximal de um nó $K$, então uma monodromia $\varphi: S_{0} \rightarrow S_{0}$ de $\phi$ é definida como no caso de $n$-nós fibrados.

Definição 4.0.2. $A$ entropia de um nó $K$, denotada por $h_{K}$, é dada por $\inf \{h(\varphi): \varphi$ é uma monodromia de alguma fibração maximal de $K\}$.

Recordemos o conceito de um nó satélite, introduzido por Schubert [21].

Assuma que $\tilde{K}$ é um nó contido em um toro sólido (padrão) $\tilde{V}$ em $S^{3}$ $\left(\tilde{V}\right.$ homeomorfo a $S^{1} \times D^{2}$ ), mas não contido em qualquer 3-bola de $\tilde{V}$. Assuma que $\hat{K}$ é um segundo nó não trivial, e seja $g: \bar{V} \rightarrow N(\hat{K})$ um difeomorfismo de $\tilde{V}$ sobre uma vizinhança tubular de $\hat{K}$, levando uma longitude de $\tilde{V}$ sobre uma longitude do nó $\hat{K}$. (Uma longitude de $\hat{K}$ é uma curva simples fechada essencial em $\partial N(\hat{K})$ que é homologicamente nula em $X(\hat{K}))$. A imagem $K=g(\tilde{K})$ é um nó não trivial que é dito nó satélite com modelo $(\tilde{V}, \tilde{K})$ e nó companheiro $\hat{K}$. Dizemos que $K$ foi composto de $\tilde{K}$ e $\hat{K}$ ou que se decompõe em $\tilde{K}$ e $\hat{K}$ (Ver [20]).

Alguns casos de decomposição vale a pena citar. Se $\tilde{K}$ é um nó toral que fica no bordo de um toro sólido $\tilde{V}_{1} \subset \tilde{V}$ tal que $\tilde{V}_{1}$ e $\tilde{V}$ tem um círculo central comum, então o nó satélite $K$ é também chamado um cabo de $\hat{K}$. Se $\tilde{K}$ é apenas o círculo central de $\tilde{V}$, então $K=\tilde{K}$, e dizemos que a decomposição satélite é trivial. Finalmente, se $\tilde{K}$ é o resultado de "amarrar" localmente o nó no círculo central de $\tilde{V}$ (isto é, em uma pequena 3-bola em $\tilde{V}$ ), então o nó satélite $K$ é o nó produto (ou soma conexa) de $\tilde{K}$ e $\hat{K}$.

Se $K$ é qualquer nó satélite com modelo $(\bar{V}, \tilde{K})$ e nó companheiro $\hat{K}$, então como em [13] denotamos o nó $\tilde{K} \subset S^{3}$ como um "quociente" $K / \tilde{K}$ de $K$ por $\tilde{K}$. Esta terminologia e notação concorda com a intuição que $\tilde{K}$ e $\hat{K}$ são menos complicados do que $K$.

Não é difícil ver que um nó $K$ tem uma decomposição satélite se e somente se seu exterior $X$ contém um toro incompressível que não é paralelo ao bordo. Equivalentemente, $K$ tem apenas a decomposição trivial exatamente quando $X$ é atoroidal; neste caso, $K$ é dito simples. 
Assuma que $K$ é um nó com exterior $X$ e uma subvariedade maximal fibrada não trivial $X_{0}$. Então $\partial X_{0}-\partial X$ é um conjunto finito $\tau$ (possivelmente vazio) de toros incompressíveis em $X_{0}$, nenhum dos quais é paralelo ao bordo. Como discutido em [16], cada toro $T \in \tau$ borda um toro sólido enodado $V \subset S^{3}$ contendo $K$, logo determina uma decomposição satélite não trivial de $K$.

Seja $\mathfrak{R}_{K}$ o conjunto de nós companheiros que surgem, isto é, seja $\mathfrak{R}_{K}$ o conjunto (possivelmente com repetição) de nós realizados por círculos centrais dos vários toros sólidos $V$ com $\partial V$ em $\tau$. Por [16], cada nó em $\mathfrak{R}_{K}$ tem uma subvariedade maximal fibrada. Se o próprio $K$ é uma subvariedade maximal fibrada trivial, então $\mathfrak{R}_{K}$ é definida por $\{K\}$. Como em [16], $\mathfrak{R}_{K}$ será chamado o sistema reduzido de nó de $K$. Naturalmente, se $K$ é fibrado, então seu sistema reduzido de nó é vazio.

Formalizemos esta nợão:

Definição 4.0.3. Um sistema reduzido de nó é um conjunto finito $\mathfrak{R}$ (possivelmente vazio) de nós não fibrados, cada um tendo uma subvariedade maximal fibrada trivial.

A coleção de todos os sistemas reduzidos de nós é parcialmente ordenada por inclusão. Um conjunto de sistemas reduzidos de nós é limitado se existe um sistema reduzido de nó $\mathfrak{R}^{*}$ tal que $\mathfrak{R} \leq \mathfrak{R}^{*}$, para qualquer $\mathfrak{R}$ no conjunto.

Lembremos que o genus de um nó é o menor genus da superfícies de Seifert do nó.

Lema 4.0.3. Seja S uma superfície compacta, conexa e orientável, possivelmente com bordo, tendo característica de Euler negativa. O número de classes conjugadas topológicas de homeomorfismos periódicos de $S$ é finito. Também, o número de classes conjugadas topológicas de homeomorfismos pseudo-Anosov de $S$ tendo dilatação limitada é finito. 
A primeira parte do enunciado do lema 4.0 .3 pode ser provado usando [2] (Teorema 1) junto com resultados sobre espaço Teichmüller. [8]

A segunda afirmação do Lema 4.0.3 segue imediatamente de dois resultados conhecidos:

(1) o número de classes conjugadas de homeomorfismos pseudo-Anosov de $S$ tendo dilatação fixa é limitado, uma afirmação de Thurston em [25] que pode ser provada usando partições de Markov (ver [26]);

(2) o conjunto de dilatações de todos os homeomorfismos pseudo- Anosov de $S$ é um subconjunto discreto de $\mathbb{R}$ (ver [6], por exemplo).

Como definido anteriormente, um enlaçamento é uma união finita $L$ de nós, disjuntos dois a dois em $S^{3}$. Dois enlaçamentos $L_{1}$ e $L_{2}$ são equivalentes se existe um difeomorfismo $f: S^{3} \rightarrow S^{3}$ tal que $f\left(L_{1}\right)=L_{2}$. Enlaçamentos equivalentes, assim como nós equivalentes, são vistos como o mesmo. Um enlaçamento é trivial se ele é composto de nós triviais localizados no interior de 3-bolas disjuntas duas a duas. O exterior de um enlaçamento $L$ é o fecho $X(L)$ de $S^{3}$ menos uma vizinhança tubular de $L$.

Observação 4.0.2. Se $f$ é um homeomorfismo que satisfaz a conclusão da Proposição 1.0.5, então diremos que $f$ está na forma canônica. Para cada componente $f_{i}, 1 \leq i \leq m$, seja $\lambda_{i}$ a dilatação de $f_{i}$ se a componente é pseudo-Anosov; se $f_{i}$ é periódica, então seja $\lambda_{i}=1$. Por [9], EGR $\left(f_{\sharp}\right)=\log \max \left\{\lambda_{1}^{1 / k_{1}}, \ldots, \lambda_{m}^{1 / k_{m}}\right\}$ $=h(f)$ (Isto está provado em [9] quando $f$ é pseudo-Anosov. Os outros casos seguem usando [1] (Teorema 4) juntamente com o fato de que qualquer Dehn twist tem entropia topologica zero).

Lema 4.0.4. Seja $L=K_{1} \cup \ldots \cup K_{n}$ um enlaçamento, e $\hat{K}_{1}, \ldots, \hat{K}_{n}$ nós não triviais. Assuma que $M$ é a 3-esfera homológica obtida colando os exteriores $X\left(\hat{K}_{1}\right), \ldots, X\left(\hat{K}_{n}\right)$ a $X(L)$ ao longo dos bordos, identificando um meridiano (respectivamente, longitude) de $\hat{K}_{i}$ com uma longitude (respectivamente meridiano) de $K_{i}, i=1, \ldots, n$. Se $M=S^{3}$, então o enlaçamento $L$ é trivial. 
Demonstração: Seja $N(L)=N\left(K_{1}\right) \cup \ldots \cup N\left(K_{n}\right)$ uma vizinhança tubular de $L$. Considere a sequência $M_{0}=S^{3}, M_{1}, \ldots, M_{n}=M$ de 3-esferas homológicas definidas indutivamente: $M_{i+1}$ é obtido de $M_{i}$ removendo o interior de $N\left(K_{i}\right)$ de $M_{i}$ e substituí-lo por $X\left(\hat{K}_{i}\right)$ (colando os bordos da maneira prescrita pelo enunciado do lema). Note que o restante das componentes $N\left(K_{i+1}\right), \ldots, N\left(K_{n}\right)$ de $N(L)$ aparecem em $M_{i}$. Também, uma longitude de qualquer $K_{j}$ em $S^{3}$, $i<j \leq n$, continua uma longitude do nó em $M_{i+1}$.

Pelo Teorema de J.W.Alexander [20](pág.107) qualquer toro mergulhado em $S^{3}$ borda um toro sólido no mínimo em um lado.

Suponha que alguma componente de $L$, por exemplo $K_{1}$, após re-rotular é não trivial. Como cada lado do toro mergulhado $T_{1}=\partial N\left(K_{1}\right)$ em $M_{1}$ borda o exterior de um nó não trivial, então $M_{1} \neq S^{3}$. Agora considere o toro mergulhado $T_{2}=\partial N\left(K_{2}\right)$ em $M_{2}$. Por um lado, $T_{2}$ borda o exterior $X\left(\hat{K}_{2}\right)$. Se o outro lado de $T_{2}$ for um toro sólido, então a 3-esfera homológica $M_{1}$ seria uma união de dois toros sólidos colados ao longo de seus bordos e logo seria um espaço lenticular. Como $S^{3}$ é a única 3-esfera homológica que é também um espaço lenticular, $M_{1}$ seria $S^{3}$, que nós sabemos que não é o caso. Logo $M_{2} \neq S^{3}$. Continuando dessa maneira, eventualmente concluímos que $M_{n} \neq S^{3}$, uma contradição. Portanto, cada componente do enlaçamento $L$ deve ser trivial.

Provemos agora por indução sobre $n$ que as componentes de $L$ podem ser separadas por 3-bolas disjuntas. Se $n=1$, então não há nada a provar. Assuma que a afirmação é verdadeira quando $n<N$, e suponha que $L$ é um enlaçamento satisfazendo as hipóteses do lema e tendo exatamente $N$ componentes $K_{1}, \ldots, K_{N}$. Seja $D$ um 2-disco em $S^{3}$ com bordo $K_{1}$. Se alguma outra componente de $L$, por exemplo $K_{2}$, após re-rotulada, não pode ser modificada por uma isotopia de forma a não tocar $D$, então assumimos que $K_{2}$ fica no toro sólido $S^{3}-i n t N\left(K_{1}\right)$ mas não está contida em nenhuma 3-bola do toro sólido. $\operatorname{Em} M_{1}=S^{3}$ a componente $K_{2}$ aparece como um nó satélite com nó companheiro $\hat{K}_{2}$; em particular, é não trivial em $S^{3}$. Segue que $M_{2}$ será alguma 3-esfera homológica diferente de $S^{3}$, e como 
no argumento anterior, $M_{N}$ não será $S^{3}$, uma contradição. Portanto, o 2-disco $D$ pode ser encontrado no exterior de $K_{2} \cup \ldots \cup K_{N}$, e assim $K_{1}$ pode ser envolvido por uma 3-bola que é disjunta de $K_{2} \cup \ldots \cup K_{N}$. O argumento no parágrafo anterior mostra que a 3-esfera homológica obtida colando $X\left(\hat{K}_{2}\right), \ldots, X\left(\hat{K}_{N}\right)$ ao exterior de $K_{2} \cup \ldots \cup K_{N}$ na maneira prescrita pelo lema é $S^{3}$. Pela hipótese de indução, o subenlaçamento $K_{2} \cup \ldots \cup K_{N}$ é trivial. Como $K_{1}$ pode ser separado do subenlaçamento, $L$ é trivial.

Teorema 4.0.3. Existe apenas um número finito de nós distintos tendo um dado genus, um sistema reduzido de nó e entropia menor que um dado limite.

Demonstração: Seja $\Re^{*}$ algum sistema reduzido de nó não-vazio, e seja $B$ uma constante positiva. É suficiente provar a seguinte afirmação:

"A coleção $\mathfrak{C}$ de nós distintos $K$ tal que $\mathfrak{R}_{K} \leq \mathfrak{R}^{*}$ e $\max \left\{\right.$ genus $\left.(K), h_{K}\right\} \leq B$ é finita." (*)

Podemos estabelecer a validade de $\left({ }^{*}\right)$ provando o seguinte enunciado para cada $m \geq 0$ :

"A coleção $\mathfrak{C}_{m}$ de nós distintos $K$ tal que $\mathfrak{R}_{K} \leq \mathfrak{R}^{*}, h_{K} \leq B$ e genus $(K)=m$ é finita." $(*)_{m}$

Vamos provar por indução sobre $m$.

(a) Caso $m=0$. Se genus $(K)=0$ ( $K$ borda um disco), então $K$ é trivial. Logo a afirmação $(*)_{0}$ é obviamente verdadeira.

(b) Caso $0<m<M$. Assuma que $(*)_{m}$ é verdadeira para todos os valores de $m$ menores que $M$. Se a subvariedade maximal fibrada trivial de um nó $K \in \mathfrak{C}_{m}$ é trivial, então $K$ deve estar contida em $\mathfrak{R}^{*}$, um conjunto finito. Portanto, é suficiente considerar apenas aqueles nós $K \in \mathfrak{C}_{m}$ com subvariedade maximal fibrada não trivial. Podemos considerar (após isotopia) que qualquer monodromia $\varphi: S_{0} \rightarrow S_{0}$ para ụma fibração maximal de um tal nó tem uma componente $\left.\varphi\right|_{S_{1}}: S_{1} \rightarrow S_{1}$ tal que $S_{1} \cap \partial X(K) \neq \emptyset$. Segue facilmente pelos argumentos de [21] ou pela prova do [16] (Lema 1.3) que $S_{1}$ pode ser estendida a 
uma superfície de Seifert de genus mínimo para $K$ através da colagem apropriada de superfícies (Seifert) ao longo de $\partial S_{1}$, uma superfície para cada componente de $\partial S_{1}-S_{1} \cap \partial X(K)$. Como o genus de $S_{1}$ mais o número de componentes de $\partial S_{1}-S_{1} \cap \partial X(K)$ é claramente limitado por $M$, o número de possibilidades para $S_{1}$ a menos de difeomorfismo é finito. Usando resultados do Capítulo 3, vemos que $h\left(\left.\varphi\right|_{S_{1}}\right) \leq h(\varphi)=h_{K} \leq B$. Consequentemente, se $\left.\varphi\right|_{S_{1}}$ é pseudo-Anosov, então sua dilatação é limitada por $e^{B}$. Pelo Lema 4.0.3 o número de possibilidades a menos de isotopia e conjugação topológica para $\left.\varphi\right|_{S_{1}}$, é finito quando for pseudoAnosov ou periódica. Logo o número de possibilidades para o "mapping torus" $X_{1}$ de $\left.\varphi\right|_{S_{1}}$ a menos de homeomorfismo (por [19] (Teorema 6.3)) é também finito. Olhamos $X_{1}$ como uma subvariedade de $X(K)$, exterior do nó .

Para contar os nós $K \in \mathfrak{C}_{m}$, fazemos o seguinte para cada uma das possíveis variedades $X_{1}$. Se $\partial X_{1}$ é conexo, então $X_{1}$ deve ser o exterior de algum nó (fibrado) $K \in \mathfrak{C}_{m}$; de fato, pelo teorema de Gordon e Luecke [12], $K$ é unicamente determinado. Se $\partial X_{1}$ consiste de mais do que uma componente, prosseguimos em três passos:

(1) Enumere as componentes de $\partial X_{1}$ como $T_{0}, T_{1}, \ldots, T_{n}$ tomando $T_{0}=\partial X(K)$ para algum $K \in \mathfrak{C}_{m}$. Colando toros sólidos $V_{1}, \ldots, V_{n}$ a $X_{1}$ ao longo dos bordos de tal forma que o meridiano de $V_{i}$ é identificado com uma componente de $S_{i} \cap T_{i}, i=$ $1, \ldots, n$. A 3-variedade resultante $X^{\prime}$ é o exterior de um nó $K^{\prime}$. Como na prova de [7] (Proposição 2.1), $X^{\prime}$ resulta de "desplicing" do exterior de algum nó $K \in \mathfrak{C}_{m}$ ao longo do toro $T_{0}, T_{1}, \ldots, T_{n}$.

(2) O nó $K^{\prime}$ é fibrado, e sua fibra é difeomorfa a $S_{1}$ "tampada" por algum número de 2-discos. Se o genus de $S_{1}$ é positivo, então $K^{\prime}$ deve ser não trivial, e por [12] um meridiano $m_{0} \subset \partial X^{\prime}$ para $K^{\prime}$ é único a menos de isotopia. Neste caso, podemos recuperar $\left(S^{3} ; K^{\prime}, V_{1}, \ldots, V_{n}\right)$ colando um toro sólido $V_{0}$ (visto como uma vizinhança tubular de $K^{\prime}$ ) a $X^{\prime}$ ao longo dos bordos, jdentificando um meridiano de $V_{0}$ com $m_{0}$. Se o genus de $S_{1}$ é zero, então $K^{\prime}$ é um nó trivial, e $X^{\prime}$ é um toro sólido. Neste caso, existem infinitas escolhas do meridiano $m_{0}$ para o qual 
$\left(S^{3} ; K^{\prime}, V_{1}, \ldots, V_{n}\right)$ pode ser recuperado colando um toro sólido $V_{0}$ como acima. Faça uma tal escolha e realize a colagem. Os círculos centrais de $V_{1}, \ldots, V_{n}$ entāo formam um enlaçamento. $L \subset S^{3}$, o fecho de uma $n$-trança geométrica $\beta$ com eixo da trança $K^{\prime}$. Diferentes escolhas de $m_{0}$ correspondem às $n$-tranças $c^{k} \beta, k \in \mathbb{Z}$, onde $c$ é uma torção total positiva de todos os fios da trança. Veremos logo que todos, exceto 2 valores de $k$, podem ser eliminados.

(3) Para cada $i=1, \ldots, n$, selecione um nó $\hat{K}$ nāo-trivial, em $\mathfrak{C}_{1} \cup \ldots \cup \mathfrak{C}_{M-1}$, um conjunto que é finito pela hipótese de indução. Faça a seleção de tal maneira que o genus $\left(S_{1}\right)+\sum n(i) \cdot \operatorname{genus}\left(\hat{K}_{i}\right)=M$, onde $n(i)$ é o número de componentes de $T_{i} \cap S_{1}$. Também, verifique que a união de todos os sistemas reduzidos de nós de $\hat{K}_{1}, \ldots, \hat{K}_{n}$ é um subconjunto de $\mathfrak{R}^{*}$. Seja $\hat{m}_{i}, \hat{l}_{i} \subset \partial X\left(\hat{K}_{i}\right)$ um par meridiano, longitude de $\hat{K}_{i}$. Remova o interior de cada toro sólido $V_{i}$ de $S^{3}$, e substitua-o por $X\left(\hat{K}_{i}\right)$, identificando $m_{i}$ (respectivamente $\left.l_{i}\right) \subset T_{i}=\partial V_{i}$ com $\hat{l}_{i}$ (respectivamente $\left.\hat{m}_{i}\right) \subset \partial X\left(\hat{K}_{i}\right)$, onde $m_{i}$ e $l_{i}$ são, respectivamente, o meridiano e a longitude padrão para $V_{i}$. Se o genus de $S_{1}$ é positivo, então o resultado é $S^{3}$, e $K^{\prime}$ é transformado em algum $K \in \mathfrak{C}_{m}$. (Claramente, a subvariedade maximal fibrada de $K$ tem uma fibra com genus no mínimo $M$. Que o genus não é maior que $M$ é uma consequência da unicidade da subvariedade maximal fibrada [15]). Se o genus de $S_{1}$ é zero, entāo pelo Lema 4.0 .4 o resultado é $S^{3}$ se e somente se o enlaçamento $L$ no passo (2) é trivial; por [11] (Lema 3) existem no máximo duas escolhas para $m_{0}$ tal que este é o caso, e logo obtemos no máximo dois possíveis nós $K \in \mathfrak{C}_{m}$. Pela recíproca do procedimento acima não é difícil ver que todo nó em $\mathfrak{C}_{m}$ pode ser produzido pelos passos (1), (2), (3).

Como o número de escolhas em cada passo é finito, assim é $\mathfrak{C}_{m}$.

O seguinte corolário é uma consequência imediata do Teorema 4.0.3 junto com o fato anteriormente observado que o conjunto de dilatações de homeomorfismos pseudo-Anosov de uma superfície fixada é discreto em $\mathbb{R}$. 
Corolário 4.0.1. Se $K_{n}$ é uma sequência infinita de nós fibrados distintos com genus limitado, então $\lim h_{K_{n}}=\infty$.

Observação 4.0.3. À primeira vista o Corolário 4.0.1 é surpreendente. Esperavase que (após isotopia para a forma canônica) uma monodromia $\varphi$, para qualquer nó fibrado não simples $K$, produzisse infinitas monodromias correspondendo a fibrações de nós distintos variando-se o número de Dehn twists entre componentes de $\varphi$, logo seriam obtidos infinitos nós fibrados distintos com a mesma entropia. Isto é verdade, mas os nós resultantes estariam necessariamente contidos em 3 esferas homológicas não simplesmente conexas e nẫo em $S^{3}$.

Se $K$ é um nó fibrado hiperbólico e $\varphi$ é uma monodromia para uma fibração de $K$, então $\varphi$ é isotópica a um homeomorfismo pseudo-Anosov com alguma dilatação $\lambda$. Neste caso, $h_{K}=\log \lambda$.

O Corolário 4.0.1 estabelece que para qualquer sequência de nós fibrados hiperbólicos, é possível distinguir seus membros se pudermos calcular as dilatações relevantes.

Da mesma maneira, como $h_{K}=\gamma_{K}$ para qualquer nó fibrado $K$, o Corolário 4.0.1 prediz que $\gamma_{K}$ seria um invariante igualmente efetivo, mais que isso, um invariante computável. (Exemplos que demonstram a sensibilidade de $\lambda_{K}$ estão contidos em [22])

Sequências de nós fibrados hiperbólicos como acima são muitas vezes construídos de um único nó fibrado usando uma certa construção "twist" de Lyon [18] e Stallings [24]. Recordemos a construção. Seja $K$ um nó fibrado e seja $\varphi: F \rightarrow F$ uma monodromia para alguma fibração de $K$. Assuma que $C \subset F$ é uma curva fechada simples que é essencial em $F$ mas não enodada em $S^{3}$. Assuma também que $C$ tem número de enlaçamento zero com uma cópia $C^{+}$de $C$ obtida puxandose $C$ para dentro de $F$. Seja $D$ um 2-disco cujo bordo estendido através de $C^{+}$ é transverso a $K$. Defina $K_{n}$ como o nó obtido de $K$ dando $|n|$ voltas completas 
passando através de $D$, voltas à direita se $n \geq 0$ e à esquerda, caso contrário. Diremos que a sequência (duplamente infinita) de nós $\left\{K_{n}\right\}_{n \in Z}$ é obtida de $K$ pela construção "twist de Lyon- Stallings". O significado da construção é que cada um dos nós $K_{n}$ é fibrado, de fato, uma monodromia para uma fibração de $K_{n}$ pode ser obtida de uma monodromia de uma fibração de $K$ compondo com $n$ Dehn twists ao longo de $C$.

$\mathrm{Na}$ construção acima não é difícil ver que $K$ encontra o disco $D$ em no mínimo dois pontos. De [17] (Teorema 3.2) nenhum nó na sequência $\left\{K_{n}\right\}_{n \in Z}$ aparece infinitas vezes. Como todos os nós $K_{n}$ têm o mesmo genus, isto é, o de $K$, temos o seguinte resultado:

Corolário 4.0.2. Se $\left\{K_{n}\right\}_{n \in Z}$ é qualquer sequência de nós obtida de um único nó fibrado $K$ pela construção twist de Lyon-Stallings, então

$$
\lim _{n \rightarrow \infty} h_{K_{n}}=\lim _{n \rightarrow \infty} h_{-K_{n}}=\infty \text {. }
$$




\section{Referências Bibliográficas}

[1] R.L. Adler, A.G. Konheim e M.H. McAndrew, Topological entropy, Trans. Amer. Math. Soc. 114 (1965) 309-319.

[2] L. Bers, An extremal problem for quasiconformal mappings, Acta Math. 141 (1978) 73-78.

[3] S. Blank e F. Laudenbach, Isotopie des formes fermées en dimension 3, Invent. Math. 54 (1979) 103-177.

[4] R. Bowen, Entropy for group endomorphisms and homogeneous spaces, Trans. Amer. Math. Soc. 153 (1970) 401-413.

[5] R. Bowen, Entropy and the fundamental group, in: the Structure of Attractors in Dynamical Systems, Lecture Notes in Mathematics 668 (Springer, New York, 1978) 21-29.

[6] P.L. Boyland e J. Franks, Notes on dynamics of surface homeomorphisms, in: Lecture Notes of the Nonlinear Systems Laboratory Workshop on Braid Types for Periodic Orbits of Surface Homeomorphisms (1989).

[7] D. Eisenbud e W. Neumann, Three-dimensional Link Theory and Invariants of Plane Curve Singularities, Annals of Mathematics Studies 110 (Princeton University Press, Princeton, NJ, 1985).

[8] A. Fathi, F. Laudenbach e V. Poenaru, Travaux de Thurston sur les surfaces, Astérisque 66-67 (1979). 
[9] A. Fathi e M. Shub, Some dynamics of pseudo-Anosov diffeomorphisms, Astérisque 66-67 (1979) 181-207.

[10] D. Fried, Fibrations over $S^{1}$ with pseudo-Anosov monodromy, Astérisque 66-67 (1979) 251-266.

[11] D.L. Goldsmith, Symmetric fibered links, in: L. P. Neuwirth, ed., Knots, Groups, and \$3\$-Manifolds, Annals of Mathematics Studies 84 (Princeton University Press, Princeton, NJ, 1975) 3-23.

[12] C. McA. Gordon e J. Luecke, Knots are determined by their complements, J. Amer. Math. Soc. 2 (1989) 374-415.

[13] R.I. Hartley, Invertible amphicheiral knots, Math. Ann. 252 (1980) 103-109.

[14] M.W. Hirsch, Differential Topology, Springer-Verlag New York Inc. 33 (1976)

[15] O. Kakimizu, On maximal fibred submanifolds of a knot exterior, Math. Ann. 284 (1989) 515-528.

[16] O. Kakimizu, Spanning surfaces and maximal fibred submanifolds for a knot, Math. Z. 210 (1992) 207-223.

[17] M. Kovno, K. Motegi e T. Shibuya, Twisting and knot types, J. Math. Soc. Japan 44 (1992) 199-216.

[18] H. Lyon, Simple knots with unique spanning surfaces, Topology 13 (1974) 275-279.

[19] J.R. Munkres, Obstructions to the smoothing of piecewise-differentiable homeomorphisms, Ann. of Math. 72 (1960) 521-554.

[20] D. Rofsen, Knots and Links, Publish or Perish, Berkeley, CA. (1976).

[21] H. Schubert, Knoten and vollinge, Acta Math. 90 (1953) 131-286. 
[22] D.S. Silver, Growth rates of \$n\$-knots, Topology Appl. 42 (1991) 217-230.

[23] D.S. Silver, Knot invariants from topological entropy, Topology and its Applications 61 (1995) 159-177.

[24] J. Stallings, Constructions of fibered knots and links, in: Proceedings of Symposia in Pure Mathematics 32 (American Mathematical Society, Providence. RI, 1978) 55-60.

[25] W.P. Thurston, On the geometry and dynamics of diffeomorphisms of surfaces, Bull. Amer. Math. Soc. 19 (1988) 417-431.

[26] W.A. Veech, The Teichmüller geodesic flow, Ann. of Math. 124 (1986) 441530.

[27] P. Walters, An Introduction to Ergodic Theory, Springer-Verlag New York Inc. 79 (1982). 